Annales Henri Lebesgue 4 (2021) 785-809

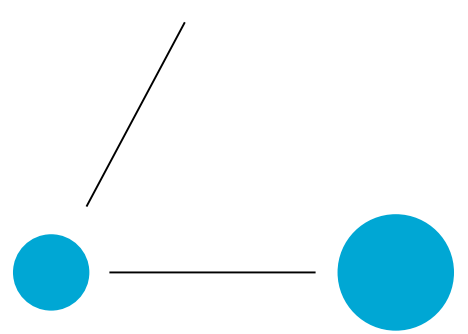

A N N A L E S HENRI LEBESGUE

\title{
EXTENSIONS OF MAXIMAL SYMPLECTIC ACTIONS ON K3 SURFACES
}

\section{EXTENSIONS D'ACTIONS SYMPLECTIQUES MAXIMALES SUR LES SURFACES K3}

Abstract. - We classify pairs $(X, G)$ consisting of a complex K3 surface $X$ and a finite group $G \leqslant \operatorname{Aut}(X)$ such that the subgroup $G_{s} \lesseqgtr G$ consisting of symplectic automorphisms is among the 11 maximal symplectic ones as classified by Mukai.

RÉsumé. - Nous classifions les paires $(X, G)$ formées d'une surface K3 complexe $X$ et d'un groupe fini $G \leqslant \operatorname{Aut}(X)$ pour lesquelles le sous-groupe $G_{s} \lesseqgtr G$ des automorphismes symplectiques appartient aux 11 sous-groupes symplectiques maximaux classifiés par Mukai.

\section{Introduction}

A (complex) K3 surface is a compact, complex manifold $X$ of dimension 2 which is simply connected and admits a nowhere degenerate holomorphic symplectic form

Keywords: K3 surface, automorphism, Mathieu group.

2020 Mathematics Subject Classification: 14J28, 14J50.

DOI: https://doi.org/10.5802/ahl.88

(*) S.B. is funded by the Deutsche Forschungsgemeinschaft (DFG, German Research Foundation) - Project-ID 286237555 - TRR 195. K. H. was partially supported by Grants-in-Aid for Scientific Research (17K14156). 
$\sigma_{X} \in \mathrm{H}^{0}\left(X, \Omega_{X}^{2}\right)$ unique up to scaling. An automorphism of a $\mathrm{K} 3$ surface is called symplectic if it leaves the 2-form invariant and non-symplectic else.

Finite groups of symplectic automorphisms of K3 surfaces were classified by Mukai up to isomorphism of groups. Namely, a group acts faithfully and symplectically on some complex K3 surface if and only if it admits an embedding into the Mathieu group $M_{24}$ which decomposes the 24 points into at least 5 orbits and fixes a point (in particular it is contained in $M_{23}$ ) [Kon98, Muk88]. This leads to a list of 11 maximal subgroups (with 5 orbits) among the subgroups of $M_{24}$ meeting these conditions. A finer classification, namely up to equivariant deformation, was obtained in [Has12]. There are 14 maximal finite symplectic group actions (see Table 4.1).

However not every automorphism of a K3 surface is symplectic. Let $X$ be a K3 surface and $G \leqslant \operatorname{Aut}(X)$ a group of automorphisms. We remark that $G$ is finite if and only if there is an ample class on $X$ invariant under $G$. Denote by $G_{s}$ the normal subgroup consisting of symplectic automorphisms. Let $G$ be finite. Then we have a natural exact sequence

$$
1 \rightarrow G_{s} \rightarrow G \stackrel{\rho}{\rightarrow} \mu_{n} \rightarrow 1
$$

where $n \in\{k \in \mathbb{N} \mid \varphi(k) \leqslant 20\}$ and $\varphi$ is the Euler totient function. The homomorphism $\rho$ is defined by $g^{*} \sigma_{X}=\rho(g) \cdot \sigma_{X}$. In the present paper, we classify finite groups $G$ of automorphisms of K3 surfaces, under the condition that $G_{s}$ is among the 11 maximal groups and $G_{s} \lessgtr G$. As it turns out, this forces the underlying K3 surface $X$ to have maximal Picard number 20, i.e. it is a singular K3 surface. In particular it has infinite automorphism group. Moreover, those K3 surfaces (with $G$ ) are rigid (i.e. not deformable). Let $(X, G)$ and $\left(X^{\prime}, G^{\prime}\right)$ be two pairs of K3 surfaces with a group of automorphisms. They are called isomorphic if there is an isomorphism $f: X \rightarrow X^{\prime}$ with $f G f^{-1}=G^{\prime}$.

Theorem 1.1. - Let $X$ be a $K 3$ surface and $G \leqslant \operatorname{Aut}(X)$ a maximal finite group of automorphisms such that the symplectic part $G_{s}$ is isomorphic to one of the 11 maximal groups and $G_{s} \lesseqgtr G$. Then the pair $(X, G)$ is isomorphic to one of the 42 pairs listed in Section 6.

The representations of the groups $G$ on the K3 lattices $\Lambda \cong \mathrm{H}^{2}(X, \mathbb{Z})$ are given in the ancillary file to [BH19] on arXiv.

The proof goes via a classification, up to conjugacy, of suitable finite subgroups of the orthogonal group of the K3 lattice. Then the strong Torelli type theorem [P $\breve{S}$ 71, BR75] and the surjectivity of the period map [Tod80] abstractly provide the existence and uniqueness of the pairs $(X, G)$.

Note that a K3 surface admitting a non-symplectic automorphism of finite order must be projective [Nik79a, Theorem 3.1]. Thus, at least in principle, it is possible to find projective models of the K3 surfaces and the automorphisms. For 25 out of the 42 pairs $(X, G)$ we list explicit equations in Section 6. Using the Torelli-type theorem Kondo proved in [Kon99] that the maximal order of a finite group of automorphisms of a K3 surface equals 3840. Section 7 is devoted to deriving its equations for the first time.

For the full story of symplectic groups of automorphisms we recommend the excellent survey [Kon18]. Non-symplectic automorphisms of prime order are treated 
in [AST11]. In [Fra11], a similar classification with different methods is carried out, albeit under the restrictive condition that $G=G_{s} \times \mu_{2}$ and the action by $\mu_{2}$ has fixed points. Note that the author misses cases $\mathbf{7 0 d}$ and $\mathbf{7 6 a}$ (see Section 6).

Remark 1.2. - Let $(X, G)$ be as in Theorem 1.1. It turns out that the nonsymplectic part $G / G_{s} \cong \mu_{n}$ is of even order and the pair $(X, G)$ is determined up to isomorphism already by $G_{s}$ and any involution in $G / G_{s}$. See Section 5 for details.

\section{Open problems}

We close this section with some interesting problems concerning groups of automorphisms of K3 surfaces.

(1) Find the remaining 17 missing equations among the $42 \mathrm{~K} 3$ surfaces and their automorphisms.

(2) Give generators of the full automorphism group of the corresponding K3 surfaces. Since a Conway chamber in the nef cone of this surface has large symmetry, chances are that one can find a nice generating set for the automorphism group.

(3) Find a projective model of the K3 surface with a linear action by $M_{22}$ in characteristic 11. Its existence is proven by Kondō [Kon06] using the crystalline Torelli type theorem.

(4) Use the present classification to study finite groups of automorphisms of Enriques surfaces beyond the semi-symplectic case [MO14].

Finding equations for the surface is often much easier than for the automorphisms. Should you find equations or relevant publications on one of the surfaces treated here, please notify the first author. We will update the arXiv version of this paper with your findings.

\section{Acknowledgements}

We would like to thank the organizers of the conference Moonshine and K3 surfaces in Nagoya in 2016 where the idea for this work was born. The first author would like to thank the University of Tokyo and Keiji Oguiso for their hospitality. Thanks to Matthias Schütt for encouragement and discussions. We warmly thank Cédric Bonnafé, Noam Elkies, Hisanori Ohashi and Alessandra Sarti for sharing explicit models of symmetric K3 surfaces with us. We also thank the anonymous referee for carefully reading our manuscript and suggesting many improvements.

\section{Lattices}

In this section we recall the basics on integral lattices (equivalently quadratic forms) and fix notation. The results are found in [CS99, Nik79b]. 
A lattice consists of a finitely generated free $\mathbb{Z}$-module $L$ and a non-degenerate integer valued symmetric bilinear form

$$
\langle\cdot, \cdot\rangle: L \times L \rightarrow \mathbb{Z}
$$

Given a basis $\left(b_{1}, \ldots, b_{n}\right)$ of $L$, we obtain the Gram matrix $Q=\left(\left\langle b_{i}, b_{j}\right\rangle\right)_{1 \leqslant i, j \leqslant n}$. The determinant $\operatorname{det} Q$ is independent of the choice of basis and called the determinant of the lattice $L$; it is denoted by $\operatorname{det} L$. We display lattices in terms of their Gram matrices. The signature of a lattice is the signature of its Gram matrix. We denote it by $\left(s_{+}, s_{-}\right)$where $s_{+}$(respectively $s_{-}$) is the number of positive (respectively negative) eigenvalues. We define the dual lattice $L^{\vee}$ of $L$ by $L^{\vee}=\{x \in L \otimes \mathbb{Q} \mid\langle x, L\rangle \subseteq \mathbb{Z}\} \cong \operatorname{Hom}(L, \mathbb{Z})$. The discriminant group $L^{\vee} / L$ is a finite abelian group of cardinality $|\operatorname{det} L|$. We call a lattice unimodular if $L=L^{\vee}$, and we call it even if $\langle x, x\rangle$ is even for all $x \in L$. The discriminant group of an even lattice carries the discriminant form

$$
q_{L}: L^{\vee} / L \rightarrow \mathbb{Q} / 2 \mathbb{Z}, \quad \bar{x} \mapsto\langle x, x\rangle+2 \mathbb{Z} .
$$

An isometry of lattices is a linear map compatible with the bilinear forms. The orthogonal group $O(L)$ is the group of isometries of $L$ and the special orthogonal group $\mathrm{SO}(L)$ consists of the isometries of determinant 1 . Discriminant forms are useful to describe embeddings of lattices and extensions of isometries. A sublattice $L \subseteq M$ is called primitive, if $L=(L \otimes \mathbb{Q}) \cap M$. By definition, the orthogonal complement $S^{\perp} \subseteq M$ of a (not necessarily primitive) sublattice $S$ is a primitive sublattice. For $L_{1}$ primitive and $L_{2}=L_{1}^{\perp}$ we call $L_{1} \oplus L_{2} \subseteq M$ a primitive extension. Now, suppose that $M$ is even, unimodular, then

$$
H_{M}=M /\left(L_{1} \oplus L_{2}\right) \subseteq\left(L_{1}^{\vee} / L_{1}\right) \oplus\left(L_{2}^{\vee} / L_{2}\right)
$$

is the graph of a so called glue $\operatorname{map} \phi_{M}: L_{1}^{\vee} / L_{1} \rightarrow L_{2} \vee / L_{2}$, that is, any element in $H_{M}$ is of the form $x \oplus \phi_{M}(x)$ for $x \in L_{1} \vee / L_{1}$. This isomorphism is an anti-isometry, namely, it satisfies $q_{L_{2}} \circ \phi_{M}=-q_{L_{1}}$. Conversely given such an anti-isometry $\phi$, its graph $H_{\phi}$ defines a primitive extension $L_{1} \oplus L_{2} \subseteq M_{\phi}$ with $M_{\phi}$ even, unimodular.

Given an isometry $f \in O\left(L_{1}\right)$, it induces an isometry $\bar{f} \in O\left(L_{1} \vee / L_{1}\right)$ of the discriminant group. Let $g \in O\left(L_{2}\right)$ be an isometry on the orthogonal complement. Then $f \oplus g \in O\left(L_{1} \oplus L_{2}\right)$ extends to $M$ if and only if $(\bar{f} \oplus \bar{g})\left(H_{M}\right)=H_{M}$, or equivalently, $\phi_{M} \circ \bar{f}=\bar{g} \circ \phi_{M}$.

Lemma 2.1. - Let $L \subseteq M$ be a primitive sublattice of an even unimodular lattice $M$. Set $O(M, L)=\{f \in O(M) \mid f(L)=L\}$ and $K=L^{\perp}$. If the natural map $O(K) \rightarrow O\left(K^{\vee} / K\right)$ is surjective, then the restriction map $O(M, L) \rightarrow O(L)$ is surjective. In other words: any isometry of $L$ can be extended to an isometry of $M$.

Proof. - Denote the glue map by $\phi=\phi_{M}$, and let $g \in O(K)$ be a preimage of $\phi \circ \bar{f} \circ \phi^{-1}$. Then $\phi \circ \bar{f}=\bar{g} \circ \phi$. Hence, $f \oplus g$ extends to $M$.

Let $L$ be a lattice and $G \leqslant O(L)$. We define the invariant and coinvariant lattices respectively by

$$
L^{G}=\{x \in L \mid \forall g \in G: g(x)=x\} \quad \text { and } \quad L_{G}=\left(L^{G}\right)^{\perp} .
$$


Then, by definition, $L^{G} \oplus L_{G} \subseteq L$ is a primitive extension. Two lattices are said to be in the same genus, if they become isometric after tensoring with the $p$-adics $\mathbb{Z}_{p}$ for all primes $p$ and the reals $\mathbb{R}$. A genus is denoted in terms of the Conway-Sloane symbols [CS99, Chapter 15]. For instance the genus of even unimodular lattices of signature $(3,19)$ is denoted by $\mathrm{II}_{3,19}$. In fact all lattices in this genus are isometric.

\section{K3 surfaces and the Torelli type theorem}

In this section we recall standard facts about complex K3 surfaces. All results can be found in the textbooks [BHPVdV04, Huy16].

Let $X$ be a $\mathrm{K} 3$ surface. Its second integral cohomology group $\mathrm{H}^{2}(X, \mathbb{Z})$ together with the cup product is an even unimodular lattice of signature $(3,19)$. It comes equipped with an integral weight 2 Hodge structure. Such a Hodge structure is given by its Hodge decomposition

$$
\mathrm{H}^{2}(X, \mathbb{Z}) \otimes \mathbb{C}=\mathrm{H}^{2}(X, \mathbb{C})=\mathrm{H}^{2,0}(X) \oplus \mathrm{H}^{1,1}(X) \oplus \mathrm{H}^{0,2}(X)
$$

with $\mathrm{H}^{i, j}(X)=\overline{\mathrm{H}^{j, i}}(X)$ and natural isomorphisms $\mathrm{H}^{i, j} \cong \mathrm{H}^{j}\left(X, \Omega_{X}^{i}\right)$. The corresponding Hodge numbers are $h^{2,0}=h^{0,2}=1$ and $h^{1,1}=20$. We can recover the entire Hodge structure from $\mathrm{H}^{2,0}(X)$ via $\mathrm{H}^{0,2}(X)=\overline{\mathrm{H}^{2,0}(X)}$ and $\mathrm{H}^{1,1}(X)=$ $\left(\mathrm{H}^{2,0}(X) \oplus \mathrm{H}^{0,2}(X)\right)^{\perp}$.

The transcendental lattice of a $\mathrm{K} 3$ surface is defined as the smallest primitive sublattice $T_{X}$ of $\mathrm{H}^{2}(X, \mathbb{Z})$ such that $T_{X} \otimes \mathbb{C}$ contains the period $\mathrm{H}^{2,0}(X)=\mathbb{C} \sigma_{X}$. By the Lefschetz theorem on $(1,1)$-classes, the Néron-Severi lattice $\mathrm{NS}_{X}$ of a K3 surface is given by $\mathrm{H}^{1,1}(X) \cap \mathrm{H}^{2}(X, \mathbb{Z})$. Note that $\mathrm{NS}_{X}$ and $T_{X}$ can be degenerate [Nik79a, (3.5)]. But if $X$ is projective, then they are (non-degenerate) lattices of signatures $(1, \rho-1)$ and $(2,20-\rho)$ respectively, and we have $\mathrm{NS}_{X}=T_{X}^{\perp}$.

As a next step we want to compare Hodge structures of different K3 surfaces. For this we fix a reference frame, namely a lattice $\Lambda \in \mathrm{II}_{3,19}$.

Definition 3.1. - A marked $K 3$ surface is a pair $(X, \eta)$ consisting of a complex $K 3$ surface $X$ and an isometry $\eta: \mathrm{H}^{2}(X, \mathbb{Z}) \rightarrow \Lambda$. We call $\eta$ a marking.

We associate a marked K3 surface $(X, \eta)$ with its period

$$
\eta_{\mathbb{C}}\left(\mathrm{H}^{2,0}(X)\right) \in \mathcal{P}_{\Lambda}:=\{\mathbb{C} \sigma \in \mathbb{P}(\Lambda \otimes \mathbb{C}) \mid\langle\sigma, \bar{\sigma}\rangle>0,\langle\sigma, \sigma\rangle=0\} .
$$

Here we extend the bilinear form on $\Lambda$ linearly to that on $\Lambda \otimes \mathbb{C}$. We call $\mathcal{P}_{\Lambda}$ the period domain. As it turns out, the concept of marking works well in families. This allows one to define the moduli space $\mathcal{M}_{\Lambda}$ of marked K3 surfaces and a period map

$$
\mathcal{M}_{\Lambda} \rightarrow \mathcal{P}_{\Lambda}, \quad(X, \eta) \mapsto \eta_{\mathbb{C}}\left(\mathrm{H}^{2,0}(X)\right)
$$

The period map is in fact holomorphic, and it turns out to be surjective as well (the surjectivity of the period map for K3 surfaces [Tod80]). The moduli space $\mathcal{M}_{\Lambda}$ is not very well behaved. For example it is not Hausdorff. This can be healed by taking into account the Kähler (resp. ample) cone. 
The positive cone $\mu_{X}$ is the connected component of the set

$$
\left\{x \in \mathrm{H}^{1,1}(X, \mathbb{R}) \mid\langle x, x\rangle>0\right\}
$$

which contains a Kähler class. Set $\Delta_{X}=\left\{x \in \mathrm{NS}_{X} \mid\langle x, x\rangle=-2\right\}$. An element in $\Delta_{X}$ is called a root. For $\delta \in \Delta_{X}$, either $\delta$ or $-\delta$ is an effective class by the RiemannRoch theorem. In fact the effective cone is generated by the effective classes in $\Delta_{X}$ and the divisor classes in the closure of the positive cone (i.e. $\mathrm{NS}_{X} \cap \overline{\mu_{X}}$ ). The connected components of $\mu_{X} \backslash \bigcup_{\delta \in \Delta_{X}} \delta^{\perp}$ are called the chambers. The hyperplanes $\delta^{\perp}$ for $\delta \in \Delta_{X}$ are called the walls. One of the chambers is the Kähler cone. For a root $\delta \in \Delta_{X}$, the reflection with respect to the wall $\delta^{\perp}$ is given by $r_{\delta}(x)=x+\langle x, \delta\rangle \delta$. The Weyl group is the subgroup of $O\left(\mathrm{H}^{2}(X, \mathbb{Z})\right)$ generated by the reflections $r_{\delta}$ for $\delta \in \Delta_{X}$. The action of the Weyl group on the chambers is simply transitive. So by composing the marking with an element of the Weyl group, we can ensure that any given chamber in the positive cone of $\Lambda$ corresponds to the Kähler cone.

Definition 3.2. - Let $X, X^{\prime}$ be K3 surfaces. An isometry $\phi: \mathrm{H}^{2}(X, \mathbb{Z}) \rightarrow$ $\mathrm{H}^{2}\left(X^{\prime}, \mathbb{Z}\right)$ is called a Hodge isometry if $\phi_{\mathbb{C}}\left(\mathrm{H}^{i, j}(X)\right) \subseteq \mathrm{H}^{i, j}\left(X^{\prime}\right)$ for all $i, j$. It is called effective, if it maps effective (resp. Kähler, resp. ample) classes on $X$ to effective (resp. Kähler, resp. ample) classes on $X^{\prime}$.

The following Torelli type theorem for K3 surfaces is the key tool for our classification of automorphisms.

TheOREM 3.3 ([BR75, PŠŠ71]). — Let $X$ and $X^{\prime}$ be complex K3 surfaces. Let

$$
\phi: \mathrm{H}^{2}(X, \mathbb{Z}) \rightarrow \mathrm{H}^{2}\left(X^{\prime}, \mathbb{Z}\right)
$$

be an effective Hodge isometry. Then there is a unique isomorphism $f: X^{\prime} \rightarrow X$ with $f^{*}=\phi$.

We thus obtain a Hodge theoretic characterization of the automorphism group of a K3 surface.

Corollary 3.4. - Let $X$ be a complex K3 surface. Then the image of the natural homomorphism

$$
\operatorname{Aut}(X) \rightarrow O\left(\mathrm{H}^{2}(X, \mathbb{Z})\right)
$$

consists of the isometries preserving the period and the Kähler cone.

\section{Symplectic automorphisms}

In this section we review known facts on symplectic automorphisms needed later on.

Let $X$ be a complex K3 surface. We obtain an exact sequence

$$
1 \rightarrow \operatorname{Aut}(X)_{s} \rightarrow \operatorname{Aut}(X) \stackrel{\rho}{\rightarrow} \mathrm{GL}\left(\mathbb{C} \sigma_{X}\right) .
$$

(Recall that we have $\mathbb{C} \sigma_{X}=\mathrm{H}^{0}\left(X, \Omega_{X}^{2}\right)$.) The elements of the kernel $\operatorname{Aut}(X)_{s}$ of $\rho$ are the symplectic automorphisms. An automorphism which is not symplectic is called non-symplectic. If $G \leqslant \operatorname{Aut}(X)$ is a group of automorphisms, we denote by $G_{s}$ 
the kernel of $\left.\rho\right|_{G}$ and call it the symplectic part of $G$. In order to keep the notation light, we identify $G$ and its isomorphic image in $O\left(\mathrm{H}^{2}(X, \mathbb{Z})\right)$.

Recall that if $L$ is a lattice and $G \leqslant O(L)$, then $L^{G}$ is the invariant and $L_{G}=\left(L^{G}\right)^{\perp}$ the coinvariant lattice. For the sake of completeness we give a proof of the following essential lemma.

Lemma 4.1 (cf. [Nik79a]). - Let $G_{s} \leqslant \operatorname{Aut}(X)_{s}$ be a finite group of symplectic automorphisms of some $K 3$ surface $X$. Then

(1) $T_{X} \subseteq \mathrm{H}^{2}(X, \mathbb{Z})^{G_{s}}$ and $\mathrm{H}^{2}(X, \mathbb{Z})_{G_{s}} \subseteq \mathrm{NS}_{X}$;

$(2) \mathrm{H}^{2}(X, \mathbb{Z})^{G_{s}}$ is of signature $(3, k)$ for some $k \leqslant 19$;

(3) $\mathrm{H}^{2}(X, \mathbb{Z})_{G_{s}}$ is negative definite;

(4) $\mathrm{H}^{2}(X, \mathbb{Z})_{G_{s}}$ contains no vectors of square -2 ;

(5) if $G_{s}$ is maximal (that is, $G_{s}$ is isomorphic to one of the 11 maximal finite groups of symplectic automorphisms), then $G_{s} \cong \operatorname{ker}\left(O(H) \rightarrow O\left(H^{\vee} / H\right)\right)$ where $H=\mathrm{H}^{2}(X, \mathbb{Z})_{G_{s}}$.

Proof.

(1) The elements of $G_{s}$ are all symplectic, i.e. they fix the 2 -form $\sigma_{X}$. Thus $\mathbb{C} \sigma_{X} \subseteq$ $\mathrm{H}^{2}(X, \mathbb{Z})^{G_{s}} \otimes \mathbb{C}$. By minimality of the transcendental lattice and primitivity of the invariant lattice, we get $T_{X} \subseteq \mathrm{H}^{2}(X, \mathbb{Z})^{G_{s}}$. Taking orthogonal complements yields the second inclusion.

(2) Let $\kappa^{\prime}$ be a Kähler class. Since automorphisms preserve the Kähler cone, the class $\kappa=\sum_{g \in G} g^{*} \kappa^{\prime}$ is a $G_{s}$-invariant Kähler class. Thus $\kappa,\left(\sigma_{X}+\bar{\sigma}_{X}\right) / 2$ and $\left(\sigma_{X}-\bar{\sigma}_{X}\right) /(2 i)$ span a positive definite subspace of dimension 3 of $\mathrm{H}^{2}(X, \mathbb{R})^{G_{s}}$.

(3) Recall that $H^{2}(X, \mathbb{Z})_{G_{s}}=\left(H^{2}(X, \mathbb{Z})^{G_{s}}\right)^{\perp}$, and $H^{2}(X, \mathbb{Z})$ has signature $(3,19)$. Now, use (2).

(4) As before we take a $G_{s}$-invariant Kähler class $\kappa$. If $r \in \mathrm{NS}_{X}$ is of square -2 , then either $r$ or $-r$ is effective by the Riemann-Roch theorem. Thus $\langle\kappa, r\rangle \neq 0$. Since $\mathrm{H}^{2}(X, \mathbb{Z})_{G_{s}}$ is orthogonal to $\kappa$, it cannot contain $r$.

(5) Let $g$ be an element in the kernel. Since $g$ acts trivially on $H^{\vee} / H$, it can be extended to an isometry $\widetilde{g}$ on $H^{2}(X, \mathbb{Z})$ such that $\left.\widetilde{g}\right|_{H^{\perp}}=\mathrm{id}_{H^{\perp}}$. As $H^{\perp} \otimes \mathbb{C}$ contains $\sigma_{X}$ and a Kähler class, $\widetilde{g}$ is in fact an effective Hodge isometry. The strong Torelli type theorem implies that it is induced by a symplectic automorphism. Since the coinvariant lattice $H$ is negative definite (by $(3)$ ), $O(H)$ is finite. In particular, the group $\widetilde{G}$ generated by $G_{s}$ and $g$ is a finite group. By the maximality of $G_{s}, G_{s}$ must contain $g$.

THEOREM 4.2 ([Has12]). - Let $G_{s}$ be a finite group of symplectic automorphisms of a $\Lambda$-marked K3 surface. Identify $G_{s}$ with its image in $O(\Lambda)$. Then the conjugacy class of $G_{s}$ is determined by the isometry class of the invariant lattice $\Lambda^{G_{s}}$. For maximal $G_{s}$, the invariant lattices can be found in Table 4.1, and the coinvariant lattice $\Lambda_{G_{s}}$ is uniquely determined up to isomorphism by the abstract group structure of $G_{s}$.

For maximal $G_{s}$, we have $\operatorname{rank} \Lambda^{G_{s}}=3$ and $\operatorname{rank} \Lambda_{G_{s}}=19$ [Muk88]. The key observation we take from Lemma 4.1, is that the invariant lattice is definite and so is the coinvariant lattice. Hence, the direct product $O\left(H^{2}(X, \mathbb{Z})^{G_{s}}\right) \times O\left(H^{2}(X, \mathbb{Z})_{G_{s}}\right)$ 
Table 4.1. Maximal finite symplectic groups of automorphisms

\begin{tabular}{|c|c|c|c|c|c|c|c|c|}
\hline No. & $G_{s}$ & $\# G_{s}$ & $\operatorname{det} \Lambda^{G_{s}}$ & genus of $\Lambda^{G_{s}}$ & $\Lambda^{G_{s}}$ & $\mathrm{SO}\left(\Lambda^{G_{s}}\right)$ & $\# O\left(\Lambda_{G_{s}}\right)$ & $\# O\left(q_{\Lambda_{G_{s}}}\right)$ \\
\hline 54 & $T_{48}$ & 48 & 384 & $2_{1}^{+1}, 8_{\text {II }}^{-2}, 3^{+1}$ & $\left(\begin{array}{ccc}2 & 0 & 0 \\
0 & 16 & 8 \\
0 & 8 & 16\end{array}\right)$ & $D_{6}$ & 9216 & 192 \\
\hline 62 & $N_{72}$ & 72 & 324 & $4_{7}^{+1}, 3^{+2}, 9^{+1}$ & $\left(\begin{array}{lll}6 & 0 & 3 \\
0 & 6 & 3 \\
3 & 3 & 12\end{array}\right)$ & $D_{4}$ & 20736 & 288 \\
\hline 63 & $M_{9}$ & 72 & 216 & $2_{1}^{-3}, 3^{+1}, 9^{+1}$ & $\left(\begin{array}{ccc}2 & 0 & 0 \\
0 & 12 & 6 \\
0 & 6 & 12\end{array}\right)$ & $D_{6}$ & 5184 & 72 \\
\hline 70 & $\mathfrak{S}_{5}$ & 120 & 300 & $4_{5}^{-1}, 3^{-1}, 5^{-2}$ & $\begin{array}{l}\left(\begin{array}{lll}4 & 1 & 0 \\
1 & 4 & 0 \\
0 & 0 & 20\end{array}\right) \\
\left(\begin{array}{ccc}4 & 2 & 2 \\
2 & 6 & 1 \\
2 & 1 & 16\end{array}\right)\end{array}$ & $\begin{array}{l}D_{2} \\
D_{2}\end{array}$ & 5760 & 48 \\
\hline 74 & $L_{2}(7)$ & 168 & 196 & $4_{7}^{+1}, 7^{+2}$ & $\begin{array}{l}\left(\begin{array}{lll}2 & 1 & 0 \\
1 & 4 & 0 \\
0 & 0 & 28\end{array}\right) \\
\left(\begin{array}{lll}4 & 2 & 2 \\
2 & 8 & 1 \\
2 & 1 & 8\end{array}\right)\end{array}$ & $\begin{array}{l}D_{2} \\
D_{4}\end{array}$ & 5376 & 32 \\
\hline 76 & $H_{192}$ & 192 & 384 & $4_{4}^{-2}, 8_{1}^{+1}, 3^{+1}$ & $\left(\begin{array}{lll}4 & 0 & 0 \\
0 & 8 & 0 \\
0 & 0 & 12\end{array}\right)$ & $D_{4}$ & 24576 & 128 \\
\hline 77 & $T_{192}$ & 192 & 192 & $4_{1}^{-3}, 3^{-1}$ & $\left(\begin{array}{lll}4 & 0 & 0 \\
0 & 8 & 4 \\
0 & 4 & 8\end{array}\right)$ & $D_{6}$ & 36864 & 192 \\
\hline 78 & $\mathfrak{A}_{4,4}$ & 288 & 288 & $2_{\text {II }}^{+2}, 8_{7}^{+1}, 3^{+2}$ & $\left(\begin{array}{lll}8 & 4 & 4 \\
4 & 8 & 2 \\
4 & 2 & 8\end{array}\right)$ & $D_{4}$ & 36864 & 128 \\
\hline 79 & $\mathfrak{A}_{6}$ & 360 & 180 & $4_{3}^{-1}, 3^{+2}, 5^{+1}$ & $\begin{array}{l}\left(\begin{array}{lll}2 & 1 & 0 \\
1 & 8 & 0 \\
0 & 0 & 12\end{array}\right) \\
\left(\begin{array}{lll}6 & 0 & 3 \\
0 & 6 & 3 \\
3 & 3 & 8\end{array}\right)\end{array}$ & $\begin{array}{l}D_{2} \\
D_{4}\end{array}$ & 11520 & 32 \\
\hline 80 & $F_{384}$ & 384 & 256 & $4_{1}^{+1}, 8_{2}^{+2}$ & $\left(\begin{array}{lll}4 & 0 & 0 \\
0 & 8 & 0 \\
0 & 0 & 8\end{array}\right)$ & $D_{4}$ & 49152 & 128 \\
\hline 81 & $M_{20}$ & 960 & 160 & $2_{\mathrm{II}}^{-2}, 8_{7}^{+1}, 5^{-1}$ & $\left(\begin{array}{lll}4 & 0 & 2 \\
0 & 4 & 2 \\
2 & 2 & 12\end{array}\right)$ & $D_{4}$ & 92160 & 96 \\
\hline
\end{tabular}

No. denotes the number of the group $G_{s} \leqslant O(\Lambda)$ as given in [Has12]. It is isomorphic to the corresponding group in the column $G_{s}$. See [Muk88] for the notation. The entry genus is given in Conway and Sloane's [CS99] notation. The dihedral group of order $2 k$ is denote by $D_{k}$.

is a finite group. It can be computed explicitly with the Plesken-Souvignier algorithm [PS97] as implemented for instance in PARI [PAR18]. As it turns out the groups $G \leqslant \operatorname{Aut}(X)$ we aim to classify are subgroups of this product.

\section{Non-symplectic extensions}

In this section we prove the classification. The invariant lattices of the symplectic actions play a major role. For a start we observe that the cyclic group $G / G_{s}$ acts on the invariant lattice. Indeed for $g \in G$ and $x \in \mathrm{H}^{2}(X, \mathbb{Z})^{G_{s}}, g G_{s}(x)=g(x)$ is well defined and lies in the invariant lattice since $G_{s}$ is normal in $G$ and fixes $x$. This yields a homomorphism

$$
G / G_{s} \rightarrow O\left(\mathrm{H}^{2}(X, \mathbb{Z})^{G_{s}}\right)
$$

of groups which turns out to be injective. 
Lemma 5.1. - Let $X$ be a $K 3$ surface and $G \leqslant \operatorname{Aut}(X)$ a finite group of automorphisms such that the subgroup $G_{s} \leqslant G$ of symplectic automorphisms is among the 11 maximal ones. Then the homomorphism $G / G_{s} \rightarrow O\left(\mathrm{H}^{2}(X, \mathbb{Z})^{G_{s}}\right)$ is injective and its image is a cyclic subgroup of $\mathrm{SO}\left(\mathrm{H}^{2}(X, \mathbb{Z})^{G_{s}}\right)$. In particular its order is $n \in\{1,2,3,4,6\}$.

Proof. - By our assumption $G_{s}$ is maximal. Thus, by Table $4.1, \mathrm{H}^{2}(X, \mathbb{Z})^{G_{s}}$ is of rank 3. Hence a basis of $\mathrm{H}^{2}(X, \mathbb{R})^{G_{s}}$ is given by a $G$-invariant Kähler class $\kappa$, $\left(\sigma_{X}+\bar{\sigma}_{X}\right) / 2$ and $\left(\sigma_{X}-\bar{\sigma}_{X}\right) /(2 i)$ (see the proof of Lemma 4.1). Since $G / G_{s}$ acts on $\mathrm{H}^{2,0}(X)=\mathbb{C} \sigma_{X}$ faithfully (by the definition of $G_{s}$ ), the injectivity in the statement of the lemma follows. By the same reason, $G / G_{s}$ is cyclic.

Let $g G_{s}$ be a generator of $G / G_{s}$. Then $(x-1)$ divides $\chi(x)=\operatorname{det}\left(x \mathrm{id}-\left.g\right|_{\mathrm{H}^{2}(X, \mathbb{Z})^{G_{s}}}\right)$. Since $\mathrm{H}^{2}(X, \mathbb{R})^{G_{s}}$ is actually defined over $\mathbb{Q}$ and $g$ is of finite order, $\chi(x)$ is a product of cyclotomic polynomials. Note that the eigenvectors $\sigma_{X}$ and $\bar{\sigma}_{X}$ have complex conjugate eigenvalues. Hence $\chi(x) \neq(x+1)(x-1)^{2}$. This leaves us with $\chi(x) /(x-1)$ to be one of $(x \pm 1)^{2}$ or $\Phi_{n}$ for $n \in\{3,4,6\}$. We conclude by computing the determinant from the characteristic polynomial.

Recall that via a marking we may identify $\mathrm{H}^{2}(X, \mathbb{Z})$ and $\Lambda$.

Remark 5.2. - A choice of basis turns the groups $\mathrm{SO}\left(\Lambda^{G_{s}}\right)$ into subgroups of $\mathrm{SL}\left(\mathbb{Z}^{3}\right)$. Finite subgroups of $\mathrm{SO}(3)$ are an essential building block for crystallographic groups. It is known that they are isomorphic to a subgroup of a dihedral group, or one of $(\mathbb{Z} / 2 \mathbb{Z})^{2} \rtimes C_{3} \leqslant(\mathbb{Z} / 2 \mathbb{Z})^{2} \rtimes S_{3}$ (cf. [AC91, Table I]). Up to conjugation there are exactly 3 subgroups of the second type, i.e. 3 integral representations of $(\mathbb{Z} / 2 \mathbb{Z})^{2} \rtimes C_{3}$. Since the three representations are irreducible, there is, up to homothety, a unique invariant quadratic form for each. Their gram matrices are given by

$$
\left(\begin{array}{lll}
1 & 0 & 0 \\
0 & 1 & 0 \\
0 & 0 & 1
\end{array}\right),\left(\begin{array}{lll}
2 & 1 & 2 \\
1 & 2 & 2 \\
2 & 2 & 4
\end{array}\right),\left(\begin{array}{lll}
3 & 2 & 2 \\
2 & 4 & 0 \\
2 & 0 & 4
\end{array}\right)
$$

and are of determinant $1,2^{2}$ and $2^{4}$. Obviously none of the invariant lattices in Table 4.1 is homothetic to one of these three. Thus $\mathrm{SO}\left(\Lambda^{G_{s}}\right)$ must be a (subgroup of) a dihedral group.

Part 2 of the next lemma will be used later in the proof of Proposition 5.9.

Lemma 5.3. - Let $H$ be one of the 14 symplectic fixed lattices and $g \in \mathrm{SO}(H)$ an involution. Then

(1) $\mathrm{SO}(H)$ is isomorphic to a dihedral group $D_{k}$ of order $2 k$ with $k \in\{2,4,6\}$;

(2) there is another involution $f \in \mathrm{SO}(H)$ in the centralizer of $g$ with $\operatorname{det} f \mid H_{g}$ $=-1$ where $H_{g}$ denotes the coinvariant lattice of the group generated by $g$.

Proof. - The proof of (1) is by a direct computation of $\mathrm{SO}(H)$ for each case using a computer. For the reader's entertainment we calculate No. 63 by hand. The fixed lattice is given as the orthogonal direct sum $(2) \oplus A_{2}(6)$ where $A_{2}(6)$ is a rescaled hexagonal lattice. The orthogonal group of the hexagonal lattice is that of the hexagon, i.e. it is the dihedral group $D_{6}$. Since the decomposition of a lattice into irreducible lattices is unique up to ordering, the orthogonal group preserves this 
decomposition and is isomorphic to $\{ \pm 1\} \times D_{6}$. It contains the special orthogonal group with index 2 . The restriction of the $\mathrm{SO}$ action to the hexagonal plane is by $D_{6}$. Thus it is in fact isomorphic to $D_{6}$.

For (2) fix $k \in\{2,4,6\}$ and let $g \in D_{k}$ be an involution. Then it is not hard to check, that there exists an involution $f$ different from $g$ and commuting with $g$. Now view $f$ and $g$ as elements of $\mathrm{SO}(H)$. The characteristic polynomials of both are equal to $(x-1)(x+1)^{2}$. If $\operatorname{det} f \mid H_{g}=1$, then $f \mid H_{g}=-\mathrm{id}$. This implies $f=g$, which we excluded.

Recall the exact sequence

$$
1 \rightarrow G_{s} \rightarrow G \rightarrow \mu_{n} \rightarrow 1
$$

From the proof of Lemma 5.1 we obtain that if $G_{s}$ is maximal, then $n \in\{1,2,3,4,6\}$. We want to reconstruct $G \leqslant O(\Lambda)$ knowing $G_{s}$ and $G / G_{s} \cong \mu_{n}$. In this situation one speaks of an extension of groups. We are interested not only in the group structure, but also in its action on the K3 lattice. This motivates the next definition.

Definition 5.4. - Let $L$ be a lattice, $G \leqslant O(L)$ a group of isometries and $N \leqslant G$ a normal subgroup with cyclic quotient $G / N=\langle g N\rangle$. We say that $G$ is an extension of $N$ by $\left.g\right|_{L^{N}}$ where $L^{N}$ denotes the invariant lattice of $N \leqslant O(L)$.

Remark 5.5. - In our setting $L$ is unimodular and $N$ coincides with the kernel of the natural map $O\left(L, L_{N}\right) \rightarrow O\left(L_{N}^{\vee} / L_{N}\right)$. In this case, $G \leqslant O(L)$ is uniquely determined by $N$ and $\left.g\right|_{L^{N}}$.

Before extending the group, we first have to extend single elements. We are in the luxurious position that every element extends:

Lemma 5.6 ([Has12, Theorem 5.1] ). - Let $\Lambda_{G_{s}}$ be the coinvariant lattice for one of the 11 maximal finite groups. Then the natural map

$$
\psi: O\left(\Lambda_{G_{s}}\right) \rightarrow O\left(\Lambda_{G_{s}} \vee / \Lambda_{G_{s}}\right)
$$

is surjective. In particular any isometry of $O\left(\Lambda^{G_{s}}\right)$ can be extended to an element in $O(\Lambda)$ normalizing $G_{s}$.

Remark 5.7. - One may double check the theorem as follows: first compute $O\left(\Lambda_{G_{s}}\right)$ with the Plesken-Souvignier backtracking algorithm. Then check by a direct computation that the natural map is surjective. For the reader's convenience we list the orders of the groups involved in Table 4.1. Note that by Lemma 4.1 we have $\# G_{s} \cdot \# O\left(q_{\Lambda_{G_{s}}}\right)=\# O\left(\Lambda_{G_{s}}\right)$, if and only if the natural map $\psi$ is surjective.

In general extensions of a given group of isometries are not unique, not even up to conjugacy. But we are in a particulary nice situation.

Lemma 5.8. - Let $G_{s} \leqslant O(\Lambda)$ be one of the 11 maximal symplectic groups. Let $g \in O\left(\Lambda^{G_{s}}\right)$ be an isometry. Then

(1) there is a unique extension of $G_{s}$ by $g$;

(2) if $\widetilde{g} \in O\left(\Lambda^{G_{s}}\right)$ is conjugate to $g$, then the corresponding extensions are conjugate in $O(\Lambda)$. 
Proof. - Recall that $G_{s}$ is a subgroup of the orthogonal group of the K3 lattice $\Lambda$. In particular we have a primitive extension $\Lambda^{G_{s}} \oplus \Lambda_{G_{s}} \subseteq \Lambda$. Since the K3 lattice is unimodular, this primitive extension is determined by an anti-isometry

$$
\phi: \Lambda^{G_{s} \vee} / \Lambda^{G_{s}} \longrightarrow \Lambda_{G_{s}} \vee / \Lambda_{G_{s}} .
$$

The natural map $\psi: O\left(\Lambda_{G_{s}}\right) \rightarrow O\left(\Lambda_{G_{s}} \vee / \Lambda_{G_{s}}\right), f \mapsto \bar{f}$ is surjective (Lemma 5.6). Hence, we find an $h \in O\left(\Lambda_{G_{s}}\right)$ such that $\bar{h}=\phi \circ \bar{g} \circ \phi^{-1}$. This means that $\widetilde{g}=g \oplus h$ extends to an isometry of $\Lambda$. We set $G=\left\langle G_{s}, \widetilde{g}\right\rangle$. Any other choice of $h$ is of the form $h \cdot\left(\operatorname{id}_{\Lambda^{G_{s}}} \oplus f\right)$ with $f \in \operatorname{ker} \psi \cong G_{s}$ (Lemma 4.1(5)). Then $G$ remains unchanged.

We turn to the second claim. Let $f \in O\left(\Lambda^{G_{s}}\right)$ and let $g^{f}=f^{-1} g f$ be a conjugate of $g$. Take an extension $\widetilde{g}$ of $g$ to an isometry of $\Lambda$. We can extend $f$ to an isometry $\tilde{f}=f \oplus f^{\prime}$ of $\Lambda$ as well (Lemma 2.1). Since the restriction $\left.G_{s}\right|_{\Lambda_{G_{s}}}$ is a normal subgroup of $O\left(\Lambda_{G_{s}}\right)$, conjugation by $f$ preserves $G_{s}$. Further the restriction of $\widetilde{g}^{\tilde{f}}$ to $\Lambda^{G_{s}}$ is equal to $g^{f}$. Hence, by part 1 , the extensions $G^{\tilde{f}}$ and $\left\langle\widetilde{g^{f}}, G_{s}\right\rangle$ are equal.

If $(X, G) \cong\left(X^{\prime}, G^{\prime}\right)$ are isomorphic pairs consisting of a $\Lambda$-marked K3 surface with a group of automorphisms, then $G$ and $G^{\prime}$ (viewed in $O(\Lambda)$ via the marking) are conjugate. In our case the pairs do not deform, so there is hope for the converse statement to hold.

Proposition 5.9. - Let $(X, \eta)$ and $\left(X^{\prime}, \eta^{\prime}\right)$ be marked $K 3$ surfaces and $G$ $\leqslant \operatorname{Aut}(X), G^{\prime} \leqslant \operatorname{Aut}\left(X^{\prime}\right)$ finite subgroups such that $G_{s}$ and $G_{s}^{\prime}$ are isomorphic to one of the 11 maximal groups. Suppose that $\eta G \eta^{-1}$ and $\eta^{\prime} G^{\prime} \eta^{\prime-1}$ are conjugate in $O(\Lambda)$, then there is an isomorphism $f: X \rightarrow X^{\prime}$ with $G=f^{-1} G^{\prime} f$, i.e. the pairs $(X, G)$ and $\left(X^{\prime}, G^{\prime}\right)$ are isomorphic.

Proof. - Changing the marking $\eta$ conjugates $\eta G \eta^{-1}$ in $O(\Lambda)$. To ease notation, we identify $G, G^{\prime}$ with their image in $O(\Lambda)$. In order to use the strong Torelli type Theorem, we have to produce an effective Hodge isometry conjugating $G$ and $G^{\prime}$.

Let $n$ be the order of $G / G_{s}$. We choose a primitive $n$th root of unity $\zeta \in \mathbb{C}$. Then $G / G_{s}$ comes with a distinguished generator $g G_{s}$ given by $g\left(\eta_{\mathbb{C}}\left(\sigma_{X}\right)\right)=\zeta \sigma_{X}$. And likewise $g^{\prime} G_{s}^{\prime}$. By assumption $G$ and $G^{\prime}$ are conjugate via some $f \in O(\Lambda)$. If $n=2$, then the generators $g G_{s}$ and $g^{\prime} G_{s}^{\prime}$ are unique. Otherwise $n=3,4,6$, and then $\mathrm{SO}\left(\Lambda^{G_{s}}\right)$ is a dihedral group of order 8 or 12 (Lemma 5.3). In any case there is a unique conjugacy class of order $n$. Since we can extend any conjugator of the dihedral group to an element of $O(\Lambda)$ (Lemma 2.1) preserving $G_{s}$, we may modify the conjugator $f$ in such a way that it conjugates the distinguished generators $g G_{s}$ and $g^{\prime} G_{s}^{\prime}$ as well. So after conjugation, we may assume that $G^{\prime}=G$ and further that $g^{\prime} G_{s}^{\prime}=g G_{s}$.

Suppose that $n>2$. Then the periods of $X$ and $X^{\prime}$ are uniquely determined by the distinguished generators as the (1-dimensional!) eigenspaces with eigenvalue $\zeta$ of $g \mid H(X, \mathbb{C})^{G}$, respectively $g^{\prime} \mid H\left(X^{\prime}, \mathbb{C}\right)^{G^{\prime}}$. And we are done. (Note that if $\sigma$ is an eigenvector for $\zeta \neq \pm 1$, then $\langle\sigma, \sigma\rangle=\zeta^{2}\langle\sigma, \sigma\rangle$ implies $\sigma^{2}=0$.) If $n=2$ then the eigenspace for -1 of $g \mid H(X, \mathbb{C})^{G}$ is of dimension 2. However, the period is of square zero. Thus the period is one of the two isotropic lines in the eigenspace. These correspond to the two orientations of the transcendental lattice. By Lemma 5.3 one can find an isometry $f$ of $\Lambda^{G}$ centralizing $g$ and reversing the orientation. This 
$f$ extends to an isometry of $\Lambda$ preserving $G$. Thus we have obtained a Hodge isometry conjugating $G$ and $G^{\prime}$.

Note that $\mathrm{H}^{2}(X, \mathbb{Z})^{G}$ is spanned by an ample class $l$ and likewise for $G^{\prime}$. Since our Hodge isometry conjugates $G$ and $G^{\prime}$ it maps $l$ to $l^{\prime}$ or $-l^{\prime}$. In the second case our Hodge isometry is not effective. However, we may then replace it by its negative.

Proposition 5.10. - Let $G_{s} \leqslant O(\Lambda)$ be a maximal symplectic group. There is a one to one correspondence between conjugacy classes of non trivial cyclic subgroups of $\mathrm{SO}\left(\Lambda^{G_{s}}\right)$ and isomorphism classes of pairs $\left(X, G^{\prime}\right)$ consisting of a K3 surface $X$ and $G^{\prime} \leqslant \operatorname{Aut}(X)$ a finite subgroup with $G_{s} \cong G_{s}^{\prime}<G^{\prime}$.

Proof. - It remains to show that each cyclic subgroup is actually coming from a K3 surface. To see this, choose a suitable eigenvector of $G /\left.G_{s}\right|_{\Lambda^{G_{s}}}$ as period, a generator of $\Lambda^{G}$ as Kähler class which is in fact ample since it is integral. Then use the global Torelli type theorem and surjectivity of the period map.

Remark 5.11. - Fix a maximal symplectic group $G_{s} \leqslant O(\Lambda)$. The non-symplectic extensions $G_{s}<G$ which we are classifying lie in the extension

$$
S O\left(\Lambda^{G_{s}}\right) \cdot G_{s}=O(\Lambda) \cap\left(S O\left(\Lambda^{G_{s}}\right) \times O\left(\Lambda_{G_{s}}\right)\right) .
$$

They are the subgroups $G_{s}<G \leqslant S O\left(\Lambda^{G_{s}}\right) \cdot G_{s}$ with $G / G_{s}$ cyclic.

Proof of Theorem 1.1. - We use the correspondence set up in Proposition 5.10. By Lemma 5.3, $\mathrm{SO}\left(\Lambda^{G_{s}}\right)$ is isomorphic to a dihedral group $D_{n}$ of order $2 n$ for $n \in\{2,4,6\}$. Its maximal cyclic subgroups up to conjugacy are two groups of order 2 generated by reflections and one group of order $n$ generated by a rotation. Thus for each of the 14 actions there are 3 maximal extensions leading to $42=3 \cdot 14$ cases.

It remains to derive the additional data which we provide with the tables. Let $\mu_{n} \cong\langle g\rangle \leqslant \mathrm{SO}\left(\Lambda^{G_{s}}\right)$ be a cyclic subgroup and $G$ the corresponding extension. The invariant polarization $\Lambda^{G}$ is computed as the kernel of $1-g \in \operatorname{End}_{\mathbb{Z}}\left(\Lambda^{G}\right)$ and the transcendental lattice as the orthogonal complement of $\Lambda^{G}$ in $\Lambda^{G_{s}}$.

To obtain the group structure of $G$, we construct $\Lambda$ as a primitive extension of $\Lambda^{G_{s}} \oplus \Lambda_{G_{s}}$ by calculating the corresponding glue map. Then we extend $g$ to an isometry $g \oplus h$ of $\Lambda$ as in Lemmas 2.1 and 5.8. Here the coinvariant lattices $\Lambda_{G_{s}}$ are obtained as sublattices of the Leech lattice as tabulated in [HM16] and the invariant lattices $\Lambda^{G_{s}}$ are tabulated in [Has12]. The gluings and extensions are carried out using the code developed by the first author for sageMath [Dev19]. We showcase the computation for Nos. 70a, 70b, 70c with a notebook in the ancillary files.

\section{The classification}

Using Proposition 5.10, we are ready to state the details of the classification. The tables were produced using SageMath [Dev19] and GAP [Gro19]. We denote by $\mathbb{Z} l=\Lambda^{G}$ the (primitive) invariant polarization of $G$. Then $G:=\operatorname{Aut}(X, l)$ is the full projective automorphism group. The lattice $\Lambda^{G_{s}}$ is the fixed lattice $H^{2}(X, \mathbb{Z})^{G_{s}}$ of a maximal symplectic action. The entry "glue" denotes the index $\left[\Lambda^{G_{s}}: T_{X} \oplus \mathbb{Z l}\right]$. The GAP Id [BEO02] identifies a group up to isomorphism. If equations for the pair 
$(X, G)$ are known, then we write its identifier in bold. We set $\zeta_{n}=\exp (2 \pi \sqrt{-1} / n)$, $\omega=\zeta_{3}$ and $i=\zeta_{4}$.

Remark 6.1. - We note that in all cases, except $\mathbf{6 2}$ b with $\mathbb{Z} l \cong(12)$, the exact sequence

$$
1 \rightarrow G_{s} \rightarrow G \rightarrow \mu_{n} \rightarrow 1
$$

splits. Namely, $G$ is a semidirect product of $G_{s}$ and $\mu_{n}$.

\subsection{No. 54}

$G_{s}=T_{48}$. We have $\mathrm{SO}\left(\Lambda^{G_{s}}\right) \cong D_{6}$.

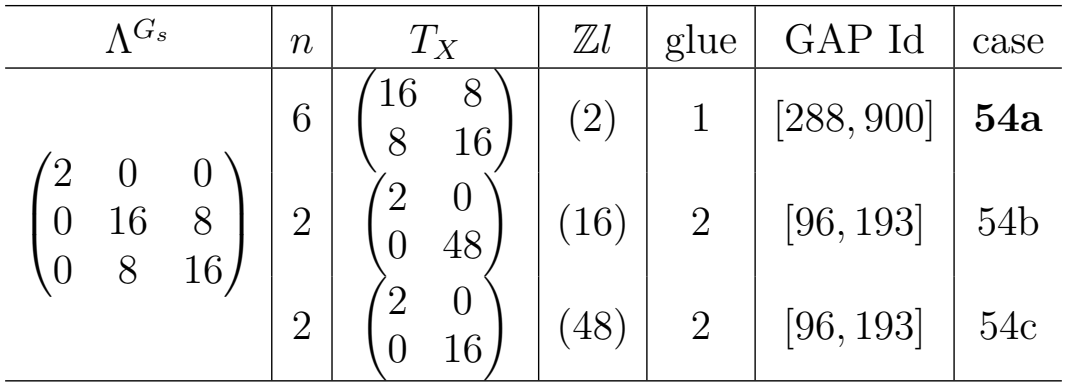

A projective model of $\mathbf{5 4 a}$ is given in [Muk88]. It is the double cover $X$ of $\mathbb{P}^{2}$ branched over the curve defined by

$$
x y\left(x^{4}+y^{4}\right)+z^{6}=0 .
$$

We have $G=G_{s} \times \mu_{6}$, where $\mu_{6}$ is generated by a lift to $X$ of $(x: y: z) \mapsto\left(x: y: \zeta_{6} z\right)$.

\subsection{No. 62}

$G_{s}=N_{72}$. We have $\mathrm{SO}\left(\Lambda^{G_{s}}\right) \cong D_{4}$.

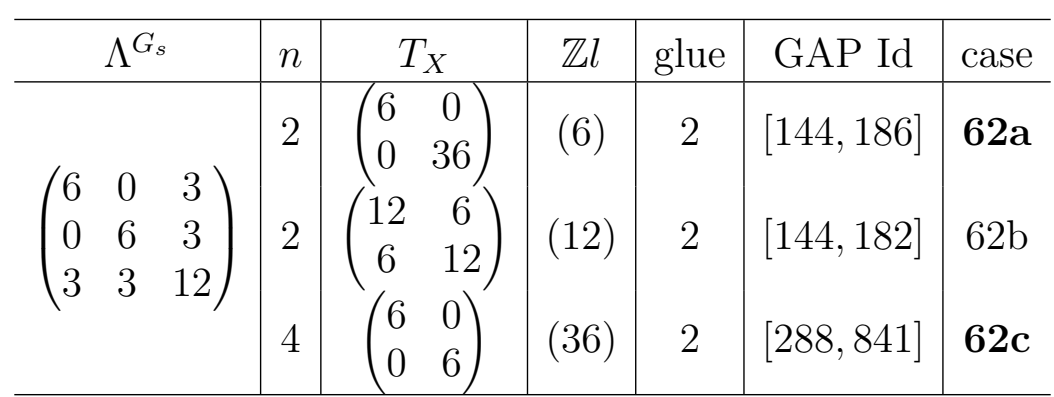

A projective model of $\mathbf{6 2 a}$ is given in [Muk88]:

$$
x_{1}^{3}+x_{2}^{3}+x_{3}^{3}+x_{4}^{3}=x_{1} x_{2}+x_{3} x_{4}+x_{5}^{2}=0 \quad \text { in } \quad \mathbb{P}^{4} .
$$

We have $G=G_{s} \times \mu_{2}$, where $\mu_{2}$ is generated by

$$
\left(x_{1}: \cdots: x_{4}: x_{5}\right) \mapsto\left(x_{1}: \cdots: x_{4}:-x_{5}\right) .
$$


A projective model of $\mathbf{6 2 c}$ in $\mathbb{P}^{5}$ together with a non-linear action of $N_{72} \times \mu_{2}$ and an invariant polarization of degree 36 is given in [MO14]:

$$
X=X_{\lambda, \mu}=\left\{\begin{array}{l}
x_{0}^{2}-\lambda x_{1} x_{2}=y_{0}^{2}-\mu y_{1} y_{2} \\
x_{1}^{2}-\lambda x_{0} x_{2}=y_{1}^{2}-\mu y_{0} y_{2} \\
x_{2}^{2}-\lambda x_{0} x_{1}=y_{2}^{2}-\mu y_{0} y_{1}
\end{array} \quad(\lambda=1+\sqrt{3}, \mu=1-\sqrt{3})\right.
$$

It remains to exhibit an element acting by a primitive $4^{\text {th }}$ root of unity on the 2-form [Oha]: the variety is constructed using the partial derivatives of the Hesse pencil

$$
z_{0}^{3}+z_{1}^{3}+z_{2}^{3}-3 \kappa z_{0} z_{1} z_{2}
$$

A linear map $g \in \mathrm{PGL}_{3}(\mathbb{C})$ preserving this pencil acts on the base as a Möbius transformation, which is denoted by the same letter $g$. By [MO14, Lemma 2.1] it induces a morphism $\tilde{g}: X_{\lambda, \mu} \rightarrow X_{g(\lambda), g(\mu)}$. Take

$$
g=\left(\begin{array}{ccc}
1 & 1 & \omega^{2} \\
1 & \omega & \omega \\
\omega & 1 & \omega
\end{array}\right) \text {. Then } g(\kappa)=\frac{\kappa+2 \omega^{2}}{\omega \kappa-1}
$$

and $g$ exchanges $\lambda$ and $\mu$. We compose $\widetilde{g}: X_{\lambda, \mu} \rightarrow X_{\mu, \lambda}$ with the map $\iota: X_{\mu, \lambda} \rightarrow X_{\lambda, \mu}$ exchanging the $x_{i}$ and $y_{i}$ to obtain the sought for automorphism of $X$, which generates $\mu_{4}$ with $G=N_{72} \rtimes \mu_{4}$. Note that $\mu_{2}$ above, which is generated by $y_{i} \mapsto-y_{i}$, is not included in $\mu_{4}$. Let $h$ be the class of hyperplane section and let $f_{\infty}$ be the class represented by the smooth elliptic curve of degree 6 defined by

$$
\operatorname{rank} A \leqslant 1, \quad \text { where } A:=\left(\begin{array}{ccc}
\mu x_{0} & x_{2}+c y_{2} & x_{1}-c y_{1} \\
x_{2}-c y_{2} & \mu x_{1} & x_{0}+c y_{0} \\
x_{1}+c y_{1} & x_{0}-c y_{0} & \mu x_{2}
\end{array}\right), c^{2}=1-\mu^{2} .
$$

Then we have $h, f_{\infty} \in \mathrm{NS}_{X}^{H}$, where $H \cong\left(C_{3}^{2} \rtimes C_{4}\right) \rtimes \mu_{4}$ is the subgroup of $G$ consisting of all linear transformations. Moreover, $3 h-f_{\infty}$ is a $G$-invariant polarization of

\begin{tabular}{|c|c|c|c|c|c|c|c|c|}
\hline \multicolumn{3}{|c|}{$\Lambda^{G_{s}}$} & $n$ & $T_{X}$ & $\mathbb{Z} l$ & glue & GAP Id & case \\
\hline \multirow{4}{*}{$\left(\begin{array}{l}2 \\
0 \\
0\end{array}\right.$} & \multirow{4}{*}{$\begin{array}{c}0 \\
12 \\
6\end{array}$} & \multirow{4}{*}{$\left.\begin{array}{c}0 \\
6 \\
12\end{array}\right)$} & 6 & $\left(\begin{array}{ll}12 & 6\end{array}\right)$ & (2) & 1 & $35]$ & 62 \\
\hline & & & & $\left(\begin{array}{ll}6 & 12\end{array}\right)$ & & & & \\
\hline & & & 2 & $\left(\begin{array}{ll}2 & 0 \\
0 & 0\end{array}\right)$ & $(12)$ & 2 & {$[144,182]$} & $63 \mathrm{~b}$ \\
\hline & & & 2 & $\left(\begin{array}{cc}2 & 0 \\
0 & 12\end{array}\right)$ & (36) & 2 & {$[144,182]$} & $63 c$ \\
\hline
\end{tabular}
degree 36 (see [MO14] for details).

\subsection{No. 63}

$G_{s}=M_{9}$. We have $\mathrm{SO}\left(\Lambda^{G_{s}}\right) \cong D_{6}$.

A projective model of $\mathbf{6 3 a}$ is given in [Muk88]. It is the double cover $X$ of $\mathbb{P}^{2}$ branched over the curve defined by

$$
x^{6}+y^{6}+z^{6}-10\left(x^{3} y^{3}+y^{3} z^{3}+z^{3} x^{3}\right)=0 .
$$


We have $G=G_{s} \rtimes \mu_{6}$, where $\mu_{6}$ is generated by the covering transformation and a lift to $X$ of $(x: y: z) \mapsto\left(\zeta_{3} x: y: z\right)$.

\subsection{No. 70}

$G_{s}=\mathfrak{S}_{5}$. We have $\mathrm{SO}\left(\Lambda^{G_{s}}\right) \cong D_{2}$ in both cases. Since the center of $G_{s}$ is trivial and there is no nontrivial outer-automorphism of $G_{s}$, we have $G=G_{s} \times \mu_{2}$ in each case.

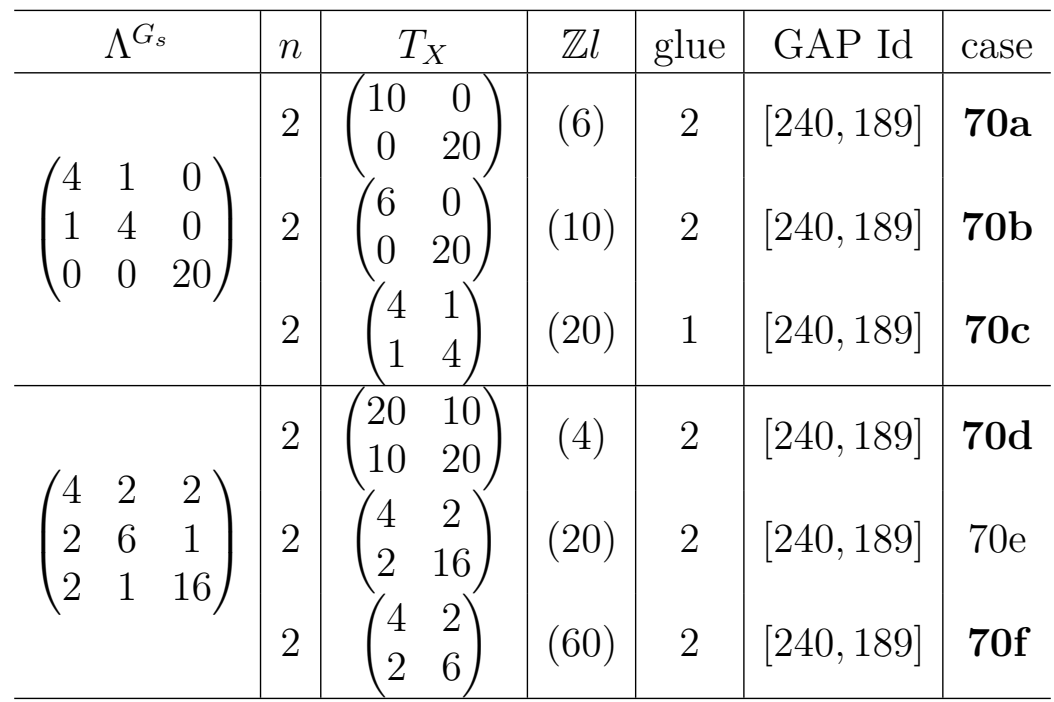

A projective model of $\mathbf{7 0 a}$ is given in [Muk88]:

$$
\sum_{i=1}^{5} x_{i}=\sum_{i=1}^{6} x_{i}^{2}=\sum_{i=1}^{5} x_{i}^{3}=0 \quad \text { in } \quad \mathbb{P}^{5},
$$

where $G=\mathfrak{S}_{5} \times \mu_{2}$ is generated by the permutations of $x_{1}, \ldots, x_{5}$ and $\left(x_{1}: \ldots: x_{5}\right.$ $\left.: x_{6}\right) \mapsto\left(x_{1}: \cdots: x_{5}:-x_{6}\right)$.

A projective model of $\mathbf{7 0 b}$ is given in [Fra11, Proposition 3.16]. Let $X$ be the minimal resolution of the double cover of $\mathbb{P}^{2}$ branched over the curve $C$ defined by $f=0$, where

$$
\begin{aligned}
f= & 2\left(x_{1}^{4} x_{2} x_{3}+x_{1} x_{2}^{4} x_{3}+x_{1} x_{2} x_{3}^{4}\right)-2\left(x_{1}^{4} x_{2}^{2}+x_{1}^{4} x_{3}^{2}+x_{1}^{2} x_{2}^{4}+x_{1}^{2} x_{3}^{4}+x_{2}^{4} x_{3}^{2}+x_{2}^{2} x_{3}^{4}\right) \\
& +2\left(x_{1}^{3} x_{2}^{3}+x_{1}^{3} x_{3}^{3}+x_{2}^{3} x_{3}^{3}\right) \\
& +\left(x_{1}^{3} x_{2}^{2} x_{3}+x_{1}^{3} x_{2} x_{3}^{2}+x_{1}^{2} x_{2}^{3} x_{3}+x_{1}^{2} x_{2} x_{3}^{3}+x_{1} x_{2}^{3} x_{3}^{2}+x_{1} x_{2}^{2} x_{3}^{3}\right)-6 x_{1}^{2} x_{2}^{2} x_{3}^{2} .
\end{aligned}
$$

Let $H \cong \mathfrak{S}_{4}$ be the subgroup of $\mathrm{PGL}_{3}(\mathbb{C})$ permuting the following four points:

$$
(1: 0: 0),(0: 1: 0),(0: 0: 1),(1: 1: 1) \text {. }
$$

Then the curve $C$ is preserved by $H$ and the following Cremona transformation (of order 5):

$$
g:\left(x_{1}: x_{2}: x_{3}\right) \mapsto\left(x_{1}\left(x_{3}-x_{2}\right): x_{3}\left(x_{1}-x_{2}\right): x_{1} x_{3}\right) .
$$

The actions of $H$ and $g$ lift to those on $X$. The group $G \cong \mathfrak{S}_{5} \times \mu_{2}$ is generated by $H$, $g$ and the covering transformation. Note that $X / \mu_{2}$ is isomorphic to the Del Pezzo 
surface of degree 5 (see [Fra11] for details). From this fact, we know the following: consider the line defined by $x_{1}=0$ in $\mathbb{P}^{2}$ and let $R_{1}$ be the strict transform of its pull-back in $X$. Then the orbit of $R_{1}\left(\right.$ in $\left.\mathrm{NS}_{X}\right)$ under the action of $G$ consists of ten curves $R_{i}(1 \leqslant i \leqslant 10)$, whose dual configuration is the Petersen graph with $R_{i} . R_{i}=-2$ and $R_{i} . R_{j} \in\{0,2\}$ for $i \neq j$ (see also [Fra11]). Hence we have

$$
R:=\sum_{i=1}^{10} R_{i}, \quad R . R=10 \cdot(-2)+2 \cdot 15 \cdot 2=40 .
$$

By construction $(X, G)$ is among the surfaces in the table. Further $R$ is invariant under $G$. Thus $R$ must lie in $\mathbb{Z} l$. This is only possible if $R=2 l$ and $l^{2}=10$. Thus $(X, G)$ is a projective model of $\mathbf{7 0 b}$.

Projective models of 70c are given in [Kon86, Type VI] and [PTvdV92]. We review the one in [PTvdV92]. Let $Y$ be the surface defined by the following equations:

$$
\sum_{i=1}^{5} x_{i}=\sum_{i=1}^{5} \frac{1}{x_{i}}=0 \quad \text { in } \quad \mathbb{P}^{4}
$$

and let $X$ be the minimal resolution of $Y$, which is a K3 surface. It follows from [Has11, Theorem 4.15] that $X$ has the same transcendental lattice as 70c. The symmetric group $\mathfrak{S}_{5}$ acts on $X$ by permutation of $x_{i}$. Moreover, the involution $\epsilon:\left(x_{i}\right) \mapsto\left(1 / x_{i}\right)$ acts on $X$. The group $G \cong \mathfrak{S}_{5} \times \mu_{2}$ is generated by $\mathfrak{S}_{5}$ and $\epsilon$.

A projective model of $\mathbf{7 0 d}$ is given in [Has11, Theorem 4.15]. Consider $Q:=\mathbb{P}^{1} \times \mathbb{P}^{1}$ defined by $\sum_{i=1}^{5} x_{i}=\sum_{i=1}^{5} x_{i}^{2}=0$ in $\mathbb{P}^{4}$. Let $X$ be the double cover of $Q$ branched over the curve defined by $\sum_{i=1}^{5} x_{i}^{4}=0$. Then $X$ is a K3 surface and $G$ is generated by the permutations of $x_{i}$ and the covering transformation. This projective model is a (degenerate) member of the family XIII in [Smi07]. The pull-back to $Q$ of the hyperplane class $h$ of $\mathbb{P}^{4}$ is of bidegree $(1,1)$, which has self intersection 2 . Hence the pull-back to $X$ of $h$ has self intersection $2 \cdot 2=4$ and is invariant under $G$. In particular it must be equal to $l$, confirming that we are indeed in case $\mathbf{7 0 d}$. Note that the linear system $|l|$ is hyperelliptic.

Projective models of $\mathbf{7 0 f}$ are given in [Kon86, Type VII] and [MO14, 1.2]. We review the one in [MO14], which is given as the minimal resolution of the singular surface

$$
\bar{X}: \quad \sum_{1 \leqslant i<j \leqslant 5} x_{i} x_{j}=\sum_{1 \leqslant i<j \leqslant 5} \frac{1}{x_{i} x_{j}}=0
$$

in $\mathbb{P}^{4}$. The symmetric group $\mathfrak{S}_{5}$ acts (non-symplectically) by permutations of the coordinates and a non-symplectic involution is given by the Enriques involution $\epsilon:\left(x_{i}\right) \mapsto\left(1 / x_{i}\right)$. The surface $\bar{X}$ has five $A_{1}$ singularities in the coordinate points. Denote by $E_{i}$ the corresponding exceptional divisors on $X$. Let $R_{i}$ be the strict transform of $\bar{X} \cap\left\{x_{i}=0\right\}$. Then the intersection numbers are given by $R_{i} \cdot C_{j}$ $=2\left(1-\delta_{i j}\right)$. An invariant polarization of degree 60 is given by $h=\sum_{i=1}^{5}\left(E_{i}+R_{i}\right)$. 


\subsection{No. 74}

$G_{s}=L_{2}(7)$. We have $\mathrm{SO}\left(\Lambda^{G_{s}}\right) \cong D_{2}, D_{4}$, respectively, as follows.

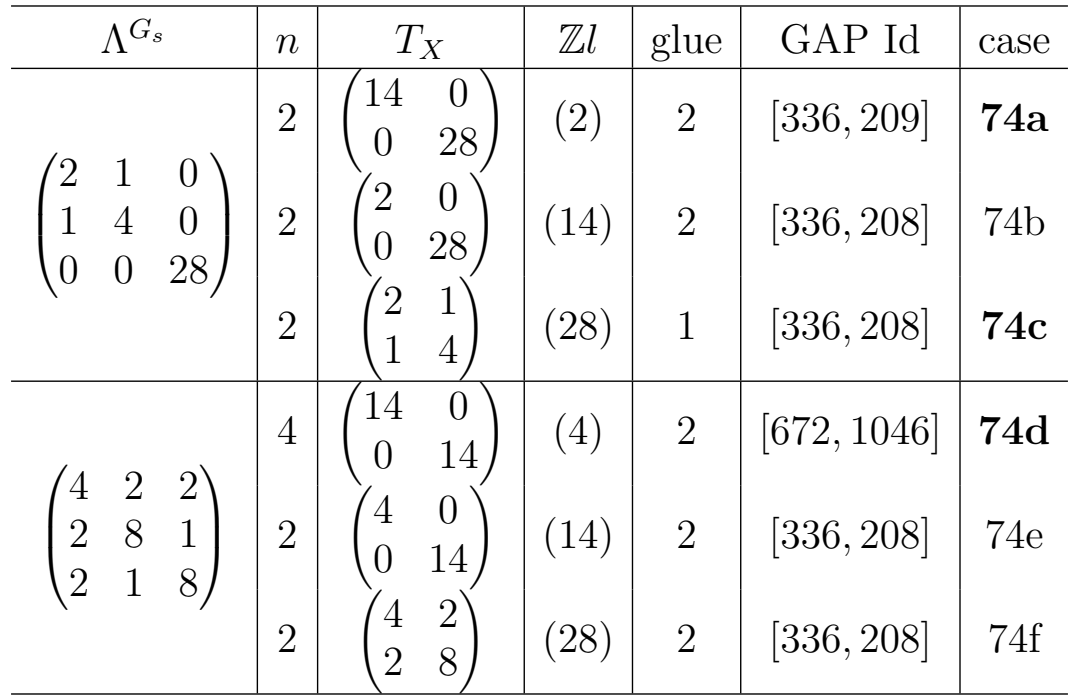

A projective model of $\mathbf{7 4 a}$ is given in [OZ02]. It is the double cover of $\mathbb{P}^{2}$ branched over the Hessian of the Klein curve defined by

$$
x^{5} z+y^{5} x+z^{5} y-5 x^{2} y^{2} z^{2}=0 .
$$

We have $G=L_{2}(7) \times \mu_{2}$, where $\mu_{2}$ is generated by the covering transformation.

A projective model of $\mathbf{7 4 c}$ is given in [Uji13] and [AST11, Appendix]. It is the universal elliptic curve over $X_{1}(7)$. We have $G=P G L_{2}\left(\mathbb{F}_{7}\right)=L_{2}(7) \rtimes \mu_{2}$.

A projective model of $\mathbf{7 4 d}$ is given in [Muk88]. Using the Klein curve with $L_{2}(7)$, it is defined by

$$
x^{3} y+y^{3} z+z^{3} x+w^{4}=0 \quad \text { in } \quad \mathbb{P}^{3} .
$$

We have $G=L_{2}(7) \times \mu_{4}$, where $\mu_{4}$ is generated by $(x: y: z: w) \mapsto(x: y: z: i w)$.

\subsection{No. 76}

$G_{s}=H_{192}$. We have $\mathrm{SO}\left(\Lambda^{G_{s}}\right) \cong D_{2}$.

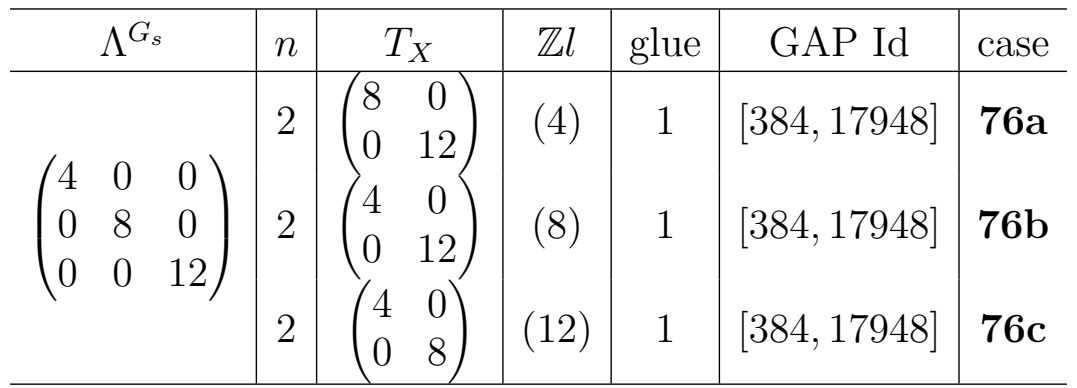

A projective model of $\mathbf{7 6} \mathbf{a}$ is given as follows (this is a (degenerate) member of the family XI in [Smi07]): consider $Q \cong \mathbb{P}^{1} \times \mathbb{P}^{1}$ defined by $\sum_{i=1}^{4} x_{i}^{2}=0$ in $\mathbb{P}^{3}$. Let $X$ be the double cover of $Q$ branched over the curve defined by $\sum_{i=1}^{4} x_{i}^{4}=0$. Then $X$ 
is a K3 surface and $G=H_{192} \times \mu_{2}$ is generated by the permutations of $x_{i}$, the sign changes of $x_{i}$, and the covering transformation. Similarly to 70d, we have $l^{2}=4$. Namely, since the pull-back to $X$ of the hyperplane class of $\mathbb{P}^{3}$ has self intersection 4 and is invariant under $G$, the pair $(X, G)$ is case $\mathbf{7 6 a}$.

A projective model of $\mathbf{7 6} \mathbf{b}$ is given in [Muk88]:

$$
x_{1}^{2}+x_{3}^{2}+x_{5}^{2}=x_{2}^{2}+x_{4}^{2}+x_{6}^{2}, \quad x_{1}^{2}+x_{4}^{2}=x_{2}^{2}+x_{5}^{2}=x_{3}^{2}+x_{6}^{2} \quad \text { in } \quad \mathbb{P}^{5} .
$$

We have $G=H_{192} \times \mu_{2}$, where $\mu_{2}$ is generated by $\left(x_{1}: \cdots: x_{6}\right) \mapsto\left(-x_{1}: x_{2}:-x_{3}\right.$ : $\left.x_{4}:-x_{5}: x_{6}\right)$.

A projective model of $\mathbf{7 6}$ c is given as a smooth surface $X$ of tri-degree $(2,2,2)$ in $\mathbb{P}^{1} \times \mathbb{P}^{1} \times \mathbb{P}^{1}[$ MO14, Example 6]:

$$
v^{2} w^{2}+u^{2} w^{2}+u^{2} v^{2}+1+i\left(u^{2}+v^{2}+w^{2}+u^{2} v^{2} w^{2}\right)=0
$$

where $(u, v, w)$ are affine coordinates on $\left(\mathbb{P}^{1}\right)^{3}$. The surface $X$ admits the following linear actions: the permutations of $(u, v, w),(u, v, w) \mapsto( \pm u, \pm v, \pm w)$, and $(u, v, w) \mapsto(i u, i v, i / w)$. Those linear actions generate $G=H_{192} \times \mu_{2}$. Here $\mu_{2}$ is generated by $(u, v, w) \mapsto(-u,-v,-w)$. The invariant polarization of degree 12 is given by $\mathcal{O}_{X}(1,1,1)$.

\subsection{No. 77}

$G_{s}=T_{192}$. We have $\mathrm{SO}\left(\Lambda^{G_{s}}\right) \cong D_{6}$.

\begin{tabular}{c|c|c|c|c|c|c}
\hline$\Lambda^{G_{s}}$ & $n$ & $T_{X}$ & $\mathbb{Z l}$ & glue & GAP Id & case \\
\hline$\left(\begin{array}{lll}4 & 0 & 0 \\
0 & 8 & 4 \\
0 & 4 & 8\end{array}\right)$ & 6 & $\left.\begin{array}{cc}8 \\
4\end{array}\right)$ & $(4)$ & 1 & {$[1152,157515]$} & $\mathbf{7 7 a}$ \\
& 2 & $\left(\begin{array}{cc}4 & 0 \\
0 & 24\end{array}\right)$ & $(8)$ & 2 & {$[384,5602]$} & $77 \mathrm{~b}$ \\
$\left(\begin{array}{cc}4 & 0 \\
0 & 8\end{array}\right)$ & $(24)$ & 2 & {$[384,5608]$} & $77 \mathrm{c}$ \\
\hline
\end{tabular}

A projective model of $\mathbf{7 7 a}$ is given in [Muk88]:

$$
x^{4}+y^{4}+z^{4}+w^{4}-2 \sqrt{-3}\left(x^{2} y^{2}+z^{2} w^{2}\right)=0 \quad \text { in } \quad \mathbb{P}^{3} .
$$

We have $G=\left(T_{24} * T_{24}\right) \rtimes\langle\tau, \sigma\rangle=T_{192} \rtimes \mu_{6}$, where $*$ denotes central product, $T_{24}$ the binary tetrahedral group, $\tau$ the involution interchanging two copies of $T_{24}$ and $\sigma$ switches the sign of $x$. 


\subsection{No. 78}

$G_{s}=\mathfrak{A}_{4,4}$. We have $\mathrm{SO}\left(\Lambda^{G_{s}}\right) \cong D_{4}$.

\begin{tabular}{|c|c|c|c|c|c|c|}
\hline$\Lambda^{G_{s}}$ & $n$ & $T_{X}$ & $\mathbb{Z} l$ & glue & GAP Id & case \\
\hline \multirow{4}{*}{$\left(\begin{array}{lll}8 & 4 & 4 \\
4 & 8 & 2 \\
4 & 2 & 8\end{array}\right)$} & 4 & $\left(\begin{array}{cc}12 & 0 \\
0 & 12\end{array}\right)$ & (8) & 2 & {$[1152,157850]$} & $78 \mathrm{a}$ \\
\hline & & & & & & \\
\hline & 2 & $\left(\begin{array}{cc}0 & 0 \\
0 & 12\end{array}\right)$ & (12) & 2 & {$[576,8654]$} & $78 \mathrm{~b}$ \\
\hline & 2 & $\left(\begin{array}{ll}8 & 4 \\
4 & 8\end{array}\right)$ & (24) & 2 & {$[576,8653]$} & $78 \mathrm{c}$ \\
\hline
\end{tabular}

A projective model of $\mathbf{7 8 a}$ is given in [Muk88]:

$$
\left(\begin{array}{ccc}
1 & 1 & 1 \\
1 & \omega & \omega^{2} \\
1 & \omega^{2} & \omega
\end{array}\right)\left(\begin{array}{l}
x^{2} \\
y^{2} \\
z^{2}
\end{array}\right)=\sqrt{3}\left(\begin{array}{c}
u^{2} \\
v^{2} \\
w^{2}
\end{array}\right) \quad \text { in } \quad \mathbb{P}^{5} .
$$

We have $G=\mathfrak{A}_{4,4} \rtimes \mu_{4}$, where $\mu_{4}$ is generated by $(x: y: z: u: v: w) \mapsto(u: v: w:$ $x: z: y)$.

\subsection{No. 79}

$G_{s}=\mathfrak{A}_{6}$. We have $\mathrm{SO}\left(\Lambda^{G_{s}}\right) \cong D_{2}, D_{4}$, respectively, as follows.

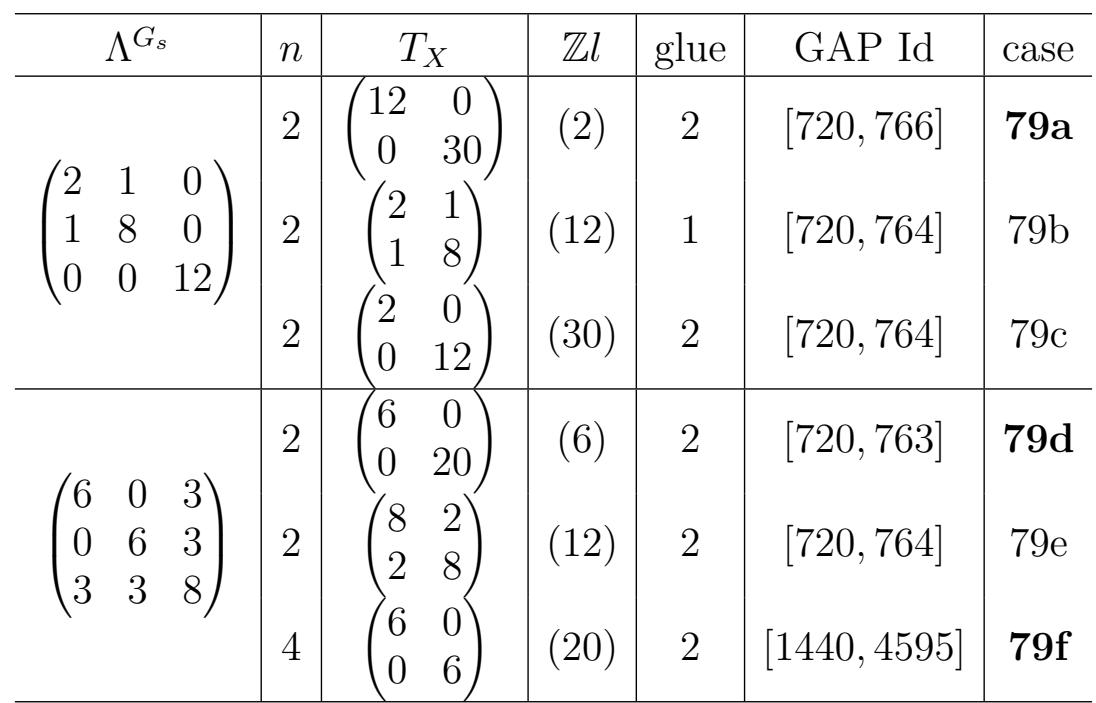

A projective model $X$ of $\mathbf{7 9 a}$ is given as follows (see also [Smi07, p. 14 (L), p. 18]). Consider the invariant curve of degree 6 by the Valentiner group in $\mathrm{GL}_{3}(\mathbb{C})$, which is defined in $\mathbb{P}^{2}$ by the following equation [Elk]:

$$
10 x^{3} y^{3}+9\left(x^{5}+y^{5}\right) z-45 x^{2} y^{2} z^{2}-135 x y z^{4}+27 z^{6}=0 .
$$

The K3 surface $X$ is defined as the double cover branched over this curve. We have $G=\mathfrak{A}_{6} \times \mu_{2}$, where $\mu_{2}$ is generated by the covering transformation. 
A projective model $X$ of $\mathbf{7 9 d}$ is given in [Muk88]:

$$
\sum_{i=1}^{6} x_{i}=\sum_{i=1}^{6} x_{i}^{2}=\sum_{i=1}^{6} x_{i}^{3}=0 \quad \text { in } \mathbb{P}^{5} .
$$

The symmetric group $\mathfrak{S}_{6}$ of degree 6 acts on $X$. The hyperplane section of $X$ has self intersection 6 and is invariant under $\mathfrak{S}_{6}$. Hence $l^{2}=6$ and $G=\mathfrak{S}_{6}=\mathfrak{A}_{6} \rtimes \mu_{2}$.

In 79f, $G / \mu_{2}=G_{s} \rtimes\left(\mu_{4} / \mu_{2}\right)$ is isomorphic to $M_{10}$ [KOZ07]. A projective model, which is same as $\mathbf{6 2 c}$, together with a (non-linear) action by $\mathfrak{A}_{6} \times \mu_{2}$ and an invariant polarization of degree 20 is given in [MO14]. The missing automorphism acting by a primitive $4^{\text {th }}$ root of unity is the one given in 62c. The invariant polarization is given by $h+f_{\infty}$ using the same notation as in 62c.

The full groups of automorphisms for 79b, 79c and 79f are calculated in [Shi16].

\subsection{No. 80}

$G_{s}=F_{384}$. We have $\mathrm{SO}\left(\Lambda^{G_{s}}\right) \cong D_{4}$.

\begin{tabular}{|c|c|c|c|c|c|c|}
\hline$\Lambda^{G_{s}}$ & $n$ & $T_{X}$ & $\mathbb{Z} l$ & glue & GAP Id & case \\
\hline \multirow{4}{*}{$\left(\begin{array}{lll}4 & 0 & 0 \\
0 & 8 & 0 \\
0 & 0 & 8\end{array}\right)$} & 4 & $\left(\begin{array}{ll}8 & 0\end{array}\right)$ & (4) & 1 & {$[1536,408544807]$} & $80 a$ \\
\hline & & & & & & \\
\hline & 2 & & (8) & 1 & {$[768,1090134]$} & $80 \mathrm{~b}$ \\
\hline & 2 & $\left(\begin{array}{cc}4 & 0 \\
0 & 16\end{array}\right)$ & (16) & 2 & {$[768,1086051]$} & $80 c$ \\
\hline
\end{tabular}

A projective model of $\mathbf{8 0 a}$ is given in [Muk88]:

$$
x^{4}+y^{4}+z^{4}+w^{4}=0 \quad \text { in } \quad \mathbb{P}^{3} .
$$

We have $G=F_{384} \rtimes \mu_{4}$, where $\mu_{4}$ is generated by $(x: y: z: w) \mapsto(i x: y: z: w)$.

A projective model of $\mathbf{8 0 b}$ [BS] is given by

$$
\begin{aligned}
& q_{1}=2 x_{2}^{2}-x_{3}^{2}-i x_{4}^{2}+i x_{5}^{2}-x_{6}^{2}=0 \\
& q_{2}=-x_{1}^{2}-i x_{2}^{2}-x_{3}^{2}+i x_{4}^{2}+2 x_{5}^{2}=0 \\
& q_{3}=-x_{1}^{2}+i x_{2}^{2}+2 x_{4}^{2}-i x_{5}^{2}-x_{6}^{2}=0
\end{aligned}
$$

with linear action generated by

$$
\left(\begin{array}{cccccc}
-1 & 0 & 0 & 0 & 0 & 0 \\
0 & -j^{2} & 0 & 0 & 0 & 0 \\
0 & 0 & 0 & 0 & j & 0 \\
0 & 0 & 0 & 0 & 0 & -j \\
0 & 0 & 0 & 1 & 0 & 0 \\
0 & 0 & j^{2} & 0 & 0 & 0
\end{array}\right) \text { and }\left(\begin{array}{cccccc}
0 & 0 & 0 & 0 & 1 & 0 \\
0 & 0 & 0 & 0 & 0 & 1 \\
0 & 0 & 0 & 1 & 0 & 0 \\
0 & 0 & 1 & 0 & 0 & 0 \\
-1 & 0 & 0 & 0 & 0 & 0 \\
0 & 1 & 0 & 0 & 0 & 0
\end{array}\right)
$$

where $j^{2}=i$. 


\subsection{No. 81}

$G_{s}=M_{20}$. We have $\mathrm{SO}\left(\Lambda^{G_{s}}\right) \cong D_{4}$.

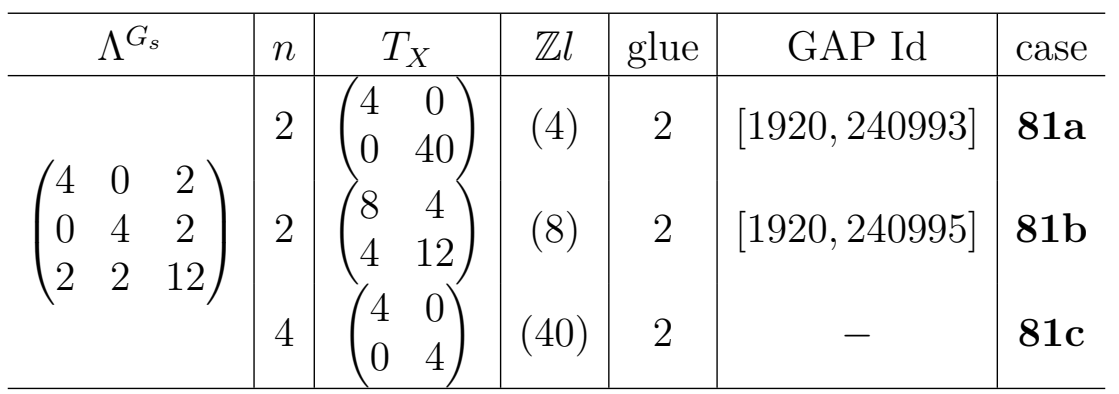

A projective model of $\mathbf{8 1 a}$ is given in [Muk88]:

$$
x^{4}+y^{4}+z^{4}+w^{4}+12 x y z w=0 \quad \text { in } \mathbb{P}^{3} .
$$

We have $G=M_{20} \rtimes \mu_{2}$, where $\mu_{2}$ is generated by $(x: y: z: w) \mapsto(y: x: z: w)$.

A projective model of $\mathbf{8 1 b}$ is given in [BS19]:

$$
\begin{aligned}
& q_{1}=x_{1}^{2}-x_{4}^{2}-\phi x_{5}^{2}+\phi x_{6}^{2}=0, \\
& q_{2}=x_{2}^{2}+\phi x_{4}^{2}+x_{5}^{2}-\phi x_{6}^{2}=0, \\
& q_{3}=x_{3}^{2}-\phi x_{4}^{2}-\phi x_{5}^{2}+x_{6}^{2}=0,
\end{aligned}
$$

where $\phi=(1+\sqrt{5}) / 2$ is the golden ratio. The group $G=G_{s} \rtimes \mu_{2}$ is generated by

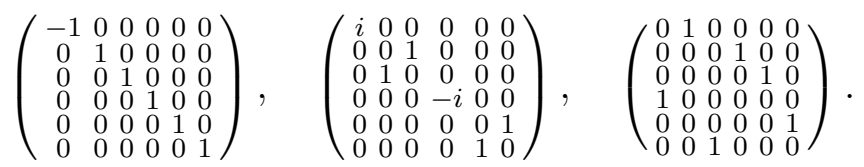

In 81c, the group $G$ has the maximal finite order 960. Its existence is proven in [Kon99] by applying lattice theory. For equations of 81c, see Section 7. The full automorphism group over $\mathbb{C}$ is calculated in [KK01]; see [Shi20] for mixed characteristic.

\section{The group of maximal order}

In this section, we give a projective model of $81 \mathrm{c}$. Let $Y$ be the surface in $\mathbb{P}^{5}$ defined by the following equations:

$$
\begin{aligned}
& f_{1}=x_{1}^{2}+x_{2}^{2}+x_{3}^{2}-x_{4}^{2} \\
& x_{3}= \\
& f_{3}=x_{1}^{2}-x_{2}^{2}
\end{aligned}
$$

The surface $Y$ has a linear action of $H_{s}=C_{2}^{4} \rtimes \mathfrak{A}_{4}$, which is generated by

$$
\begin{aligned}
\left(x_{1}: \cdots: x_{6}\right) & \mapsto\left(x_{1}: x_{2}: i x_{4}: i x_{3}: x_{6}: x_{5}\right), \\
\left(x_{1}: \cdots: x_{6}\right) & \mapsto\left(-x_{1}: x_{2}:-x_{3}: x_{4}: x_{5}: x_{6}\right), \\
\left(x_{1}: \cdots: x_{6}\right) & \mapsto\left(x_{3}: x_{4}: x_{5}: x_{6}: x_{1}: x_{2}\right) .
\end{aligned}
$$


Moreover, $Y$ has an automorphism $h$ of order 4:

$$
h:\left(x_{1}: \cdots: x_{6}\right) \mapsto\left(x_{1}: i x_{2}: x_{5}: i x_{6}: x_{3}: i x_{4}\right) .
$$

There are 16 singular points $p_{1}, \ldots, p_{16}$ of $Y$, e.g. $(0: 1: 0: 1: 0: 1) \in \mathbb{P}^{5}$. They form one orbit under the $H_{s}$-action and each of them is of type $A_{1}$. Let $\pi: X \rightarrow Y$ be the minimal resolution. Then $X$ is a K3 surface. The induced action of $H_{s}$ on $X$ is symplectic and we have $h^{*} \omega_{X}=i \omega_{X}$. Let $l^{\prime} \in \mathrm{NS}_{X}$ denote the pull-back of the class of hyperplane section of $Y$. Furthermore, let $d \in \mathrm{NS}_{X}$ denote the sum of the classes of the 16 exceptional curves of $\pi$. Then we have

$$
l:=3 l^{\prime}-d, \quad l^{2}=9 \cdot l^{\prime 2}+d^{2}=9 \cdot 8+16 \cdot(-2)=40,
$$

and

$$
\mathrm{H}^{0}(X, l) \cong\left\{s \in R_{3} \mid s\left(p_{1}\right)=\cdots=s\left(p_{16}\right)=0\right\} / I_{3} \subset R_{3} / I_{3} \cong \mathrm{H}^{0}\left(X, 3 l^{\prime}\right),
$$

where $R_{3}$ and $I_{3}$ are the homogeneous parts of degree 3 of $R:=\mathbb{C}\left[x_{1}, \ldots, x_{6}\right]$ and the defining ideal $I$ of $Y$, respectively. (Hence $I_{3}$ is spanned by $x_{i} f_{j}$ for $1 \leqslant i \leqslant 6$ and $1 \leqslant j \leqslant 3$.) We take the following basis of $\mathrm{H}^{0}(X, l) \cong \mathbb{C}^{22}$ :

$$
\begin{aligned}
\left(z_{1}, \ldots, z_{22}\right)= & \left(x_{1} x_{2} x_{3}, x_{1} x_{2} x_{4}, x_{3} x_{4} x_{5}, x_{3} x_{4} x_{6}, x_{1} x_{5} x_{6}, x_{2} x_{5} x_{6},\right. \\
& x_{1} x_{2} x_{5}, x_{1} x_{2} x_{6}, x_{1} x_{3} x_{4}, x_{2} x_{3} x_{4}, x_{3} x_{5} x_{6}, x_{4} x_{5} x_{6}, \\
& \left.x_{1}^{2} x_{2}, x_{1} x_{2}^{2}, x_{3}^{2} x_{4}, x_{3} x_{4}^{2}, x_{5}^{2} x_{6}, x_{5} x_{6}^{2}, x_{1} x_{3} x_{5}, x_{1} x_{4} x_{6}, x_{2} x_{3} x_{6}, x_{2} x_{4} x_{5}\right) .
\end{aligned}
$$

(The Riemann-Roch theorem also implies $\operatorname{dim} \mathrm{H}^{0}(X, l)=l^{2} / 2+2=22$.) The complete linear system for $l$ gives a smooth embedding of $X$ into $\mathbb{P}^{21}$ with coordinates $z_{1}, \ldots, z_{22}$. Moreover, the coordinates $z_{1}, \ldots, z_{6}$ define a non-normal model $\bar{X}$ of $X$ in $\mathbb{P}^{5}$. By using [DGPS19, Singular], one can check the following: the defining ideal of $\bar{X}$ is generated by $\left(\bar{g}^{i}\right)^{*} q$ for $0 \leqslant i \leqslant 4$, where

$$
\begin{aligned}
& q:=\left(-z_{1}^{2}+z_{2}^{2}-z_{3}^{2}-z_{4}^{2}\right) z_{5}^{2}+\left(z_{1}^{2}-z_{2}^{2}-z_{3}^{2}-z_{4}^{2}\right) z_{6}^{2}+z_{5}^{4}-z_{6}^{4}, \\
& \bar{g}:\left(z_{1}, \ldots, z_{6}\right) \mapsto\left(-i z_{2},-z_{3},-z_{5},-i z_{1},-z_{4}, z_{6}\right) .
\end{aligned}
$$

The automorphism of $\bar{X}$ (of order 5 ) induced by $\bar{g}$ is extended to an automorphism $g$ of $X$ embedded into $\mathbb{P}^{21}$, where $g$ is given by

$$
\begin{aligned}
g:\left(z_{1}: \cdots: z_{22}\right) & \mapsto\left(-i z_{2}:-z_{3}:-z_{5}:-i z_{1}:-z_{4}: z_{6}:\right. \\
i z_{12} & :-z_{10}: z_{9}:-z_{7}:-z_{8}: i z_{11}: \\
-i z_{16} & \left.: i z_{22}: z_{13}:-i z_{19}: z_{18}: i z_{21}:-i z_{20}:-i z_{15}:-z_{14}: z_{17}\right) .
\end{aligned}
$$

By a direct computation, one can check that the following Cremona transformation acts on $Y$ and induces $g$ :

$$
\left(x_{1}: \cdots: x_{6}\right) \mapsto\left(x_{3} x_{4}:-x_{2} x_{5}: x_{1} x_{2}:-i x_{3} x_{5}:-x_{5} x_{6}:-i x_{2} x_{3}\right) .
$$

The group $G_{s}$ of all symplectic automorphisms of $X$ with polarization $l$ is generated by $H_{s}$ and $g$. We have $G_{s} \cong M_{20}$. The group $G \cong M_{20} \rtimes \mu_{4}$ of all automorphisms of $X$ with polarization $l$ is generated by $G_{s}$ and $h$. 
Remark 7.1. - The motivation for this construction is as follows. Let $X$ be a K3 surface with an action of $G=M_{20} \rtimes \mu_{4}$ as in 81c. We consider a maximal proper subgroup $H_{s}$ of $G_{s}=M_{20}$ isomorphic to $2^{4} \cdot \mathfrak{A}_{4}$. From [Has12, Table 10.3], we get $\operatorname{rank} \Lambda^{H_{s}}=4$ and the genus symbol of $\Lambda^{H_{s}}$ is $2_{\mathrm{II}}^{-2}, 8_{2}^{-2}$. (In [Has12], $H_{s}$ is No. 75 and its structure is written as $4^{2} \mathfrak{A}_{4}$.) Consider the lattice $L$ of rank 4 with basis $\left(b_{1}, \ldots, b_{4}\right)$ and Gram matrix

$$
\left(\begin{array}{cccc}
4 & 0 & 0 & 0 \\
0 & 4 & 0 & 0 \\
0 & 0 & 8 & 0 \\
0 & 0 & 0 & -8
\end{array}\right) .
$$

The lattice $\bar{L}$ generated by $L$ and $\left(b_{1}+b_{2}+b_{3}\right) / 2$ is isomorphic to $\Lambda^{H_{s}}$. Consider an isometry of $h$ defined by $\left(b_{1}, \ldots, b_{4}\right) \mapsto\left(b_{2},-b_{1}, b_{3}, b_{4}\right)$, which extends to $\bar{L}$. By a lattice-theoretic argument, it follows that the action of $\mu_{4}$ on $\Lambda^{H_{s}}$ corresponds to $\langle h\rangle$ and there is an ample class $l^{\prime}$ of degree 8 giving rise to $b_{3}$. This suggests that there is a complete intersection of type $(2,2,2)$ in $\mathbb{P}^{5}$ birational to $X$, which is nothing but $Y$ above. The classes $l$ and $d$ correspond to $3 b_{3}-2 b_{4}$ and $2 b_{4}$, respectively. Indeed, calculating the orthogonal complement of $l^{\prime}$ inside the Néron-Severi lattice one finds 16 vectors (up to sign) of square $(-2)$. These give the 16 singular points of type $A_{1}$ of $Y$ and their sum is $2 b_{4}$.

\section{BIBLIOGRAPHY}

[AC91] Louis Auslander and Michael Cook, An algebraic classification of the threedimensional crystallographic groups, Adv. Appl. Math. 12 (1991), no. 1, 1-21. $\uparrow 793$

[AST11] Michela Artebani, Alessandra Sarti, and Shingo Taki, K3 surfaces with nonsymplectic automorphisms of prime order. With an appendix by Shigeyuki Kondo, Math. Z. 268 (2011), no. 1-2, 507-533. $\uparrow 787,801$

[BEO02] Hans Ulrich Besche, Bettina Eick, and Eamonn A. O'Brien, A millennium project: constructing small groups, Int. J. Algebra Comput. 12 (2002), no. 5, 623-644. 7796

[BH19] Simon Brandhorst and Kenji Hashimoto, Extensions of maximal symplectic actions on K3 surfaces, https://arxiv.org/abs/1910.05952, 2019. $\uparrow 786$

[BHPVdV04] Wolf P. Barth, Klaus Hulek, Chris A. M. Peters, and Antonius Van de Ven, Compact complex surfaces, second ed., Ergebnisse der Mathematik und ihrer Grenzgebiete. 3. Folge., vol. 4, Springer, 2004. $\uparrow 789$

[BR75] Dan Jr. Burns and Michael Rapoport, On the Torelli problem for kählerian K3 surfaces, Ann. Sci. Éc. Norm. Supér. 8 (1975), no. 2, 235-273. ^786, 790

[BS] Cédric Bonnafé and Alessandra Sarti, Private communication. $\uparrow 804$

[BS19] K3 surfaces with maximal finite automorphism groups containing $M_{20}$, https://arxiv.org/abs/1910.05955, 2019. $\uparrow 805$

[CS99] John H. Conway and Neil J. A. Sloane, Sphere packings, lattices and groups, third ed., Grundlehren der Mathematischen Wissenschaften, vol. 290, Springer, 1999. $\uparrow 787$, 789,792

[Dev19] The Sage Developers, SageMath, the Sage Mathematics Software System, 2019, (version 8.9), https://www. sagemath.org. $\uparrow 796$

[DGPS19] Wolfram Decker, Gert-Martin Greuel, Gerhard Pfister, and Hans Schönemann, SINGULAR 4-1-2 - A computer algebra system for polynomial computations, http: //www. singular .uni-kl.de, 2019. $\uparrow 806$ 
[Elk] Noam D. Elkies, Private communication. $\uparrow 803$

[Fra11] Kristina Frantzen, Classification of K3-surfaces with involution and maximal symplectic symmetry, Math. Ann. 350 (2011), no. 4, 757-791. ^787, 799, 800

[Gro19] The GAP Group, Gap - Groups, Algorithms, and Programming, 2019, version 4.10.2, https : //www.gap-system.org. $\uparrow 796$

[Has11] Kenji Hashimoto, Period map of a certain $K 3$ family with an $\mathfrak{S}_{5}$-action, J. Reine Angew. Math. 652 (2011), 1-65, With an appendix by Tomohide Terasoma. $\uparrow 800$

[Has12] _ Finite symplectic actions on the K3 lattice, Nagoya Math. J. 206 (2012), 99-153. $7786,791,792,794,796,807$

[HM16] Gerald Höhn and Geoffrey Mason, The 290 fixed-point sublattices of the Leech lattice, J. Algebra 448 (2016), 618-637. $\uparrow 796$

[Huy16] Daniel Huybrechts, Lectures on K3 surfaces, Cambridge Studies in Advanced Mathematics, vol. 158, Cambridge University Press, 2016. $\uparrow 789$

[KK01] Jonghae Keum and Shigeyuki Kondō, The automorphism groups of Kummer surfaces associated with the product of two elliptic curves, Trans. Am. Math. Soc. 353 (2001), no. 4, 1469-1487. $\uparrow 805$

[Kon86] Shigeyuki Kondō, Enriques surfaces with finite automorphism groups, Jpn. J. Math., New Ser. 12 (1986), no. 2, 191-282. $\uparrow 800$

[Kon98] _ Niemeier lattices, Mathieu groups, and finite groups of symplectic automorphisms of K3 surfaces. With an appendix by Mukai, Shigeru, Duke Math. J. 92 (1998), no. 3, 593-603. $\uparrow 786$

[Kon99] 는 The maximum order of finite groups of automorphisms of K3 surfaces, Am. J. Math. 121 (1999), no. 6, 1245-1252. $\uparrow 786,805$

[Kon06] Maximal subgroups of the Mathieu group $M_{23}$ and symplectic automorphisms of supersingular K3 surfaces, Int. Math. Res. Not. (2006), article no. 71517. $\uparrow 787$

[Kon18] _ A survey of finite groups of symplectic automorphisms of K3 surfaces, J. Phys. A, Math. Theor. 51 (2018), no. 5, article no. 053003. $\uparrow 786$

[KOZ07] Jonghae Keum, Keiji Oguiso, and De-Qi Zhang, Extensions of the alternating group of degree 6 in the geometry of K3 surfaces, Eur. J. Comb. 28 (2007), no. 2, 549-558. $\uparrow 804$

[MO14] Shigeru Mukai and Hisanori Ohashi, Finite groups of automorphisms of Enriques surfaces and the Mathieu group $M_{12}$, https://arxiv.org/abs/1410.7535, 2014. $\uparrow 787,798,800,802,804$

[Muk88] Shigeru Mukai, Finite groups of automorphisms of K3 surfaces and the Mathieu group, Invent. Math. 94 (1988), no. 1, 183-221. ^786, 791, 792, 797, 798, 799, 801, $802,803,804,805$

[Nik79a] Vyacheslav V. Nikulin, Finite groups of automorphisms of Kählerian surfaces of type K3, Tr. Mosk. Mat. O.-va 38 (1979), 75-137. $\uparrow 786$, 789, 791

[Nik79b] Integer symmetric bilinear forms and some of their geometric applications, Izv. Akad. Nauk SSSR, Ser. Mat. 43 (1979), no. 1, 111-177, 238. $\uparrow 787$

[Oha] Hisanori Ohashi, Private communication. $\uparrow 798$

[OZ02] Keiji Oguiso and De-Qi Zhang, The simple group of order 168 and K3 surfaces, Complex geometry. Collection of papers dedicated to Hans Grauert on the occasion of his 70th birthday, Springer, 2002, pp. 165-184. $\uparrow 801$

[PAR18] PARI Group, Pari/GP version 2.11.1, 2018, available from http://pari.math. u-bordeaux.fr/. $\uparrow 792$

[PS97] Wilhelm Plesken and Bernd Souvignier, Computing isometries of lattices, J. Symb. Comput. 24 (1997), no. 3-4, 327-334. $\uparrow 792$ 
[PŠŠ71] Ilya I. Pjateckiı̌-Šapiro and Igor R. Šafarevič, Torelli's theorem for algebraic surfaces of type K3, Izv. Akad. Nauk SSSR, Ser. Mat. 35 (1971), 530-572. $\uparrow 786,790$

[PTvdV92] Chris A. M. Peters, Jaap Top, and Marcel van der Vlugt, The Hasse zeta function of a K3-surface related to the number of words of weight 5 in the Melas codes, J. Reine Angew. Math. 432 (1992), 151-176. $\uparrow 800$

[Shi16] Ichiro Shimada, The automorphism groups of certain singular K3 surfaces and an Enriques surface, K3 surfaces and their moduli, Basel: Birkhäuser/Springer, 2016, pp. 297-343. $\uparrow 804$

[Shi20] _ The elliptic modular surface of level 4 and its reduction modulo 3, Ann. Mat. Pura Appl. 199 (2020), no. 4, 1457-1489. $\uparrow 805$

[Smi07] James P. Smith, Picard-Fuchs Differential Equations for Families of K3 Surfaces, https://arxiv.org/abs/0705.3658, 2007. $\uparrow 800,801,803$

[Tod80] Andrei N. Todorov, Applications of the Kähler-Einstein-Calabi-Yau metric to moduli of K3 surfaces, Invent. Math. 61 (1980), no. 3, 251-265. $\uparrow 786,789$

[Uji13] Masashi Ujikawa, The automorphism group of the singular K3 surface of discriminant 7, Comment. Math. Univ. St. Pauli 62 (2013), no. 1, 11-29. $\uparrow 801$

Manuscript received on 4th May 2020, revised on 7 th October 2020 ,

accepted on 23rd November 2020.

Recommended by Editor S. Cantat.

Published under license CC BY 4.0.

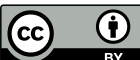

This journal is a member of Centre Mersenne.

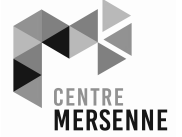

Simon BRANDHORST

Fakultät für Mathematik und Informatik,

Universität des Saarlandes,

Campus E2.4, 66123 Saarbrücken, (Germany)

brandhorst@math.uni-sb.de

Kenji HASHIMOTO

Graduate School of Mathematical Sciences,

The University of Tokyo,

3-8-1 Komaba, Maguro-ku, Tokyo,

153-8914, (Japan)

kenji.hashimoto.math@gmail.com 